\title{
Hadronic light-by-light contribution to the muon $g-2$ from holographic QCD with massive pions
}

\author{
Josef Leutgeb $\odot$ and Anton Rebhan 1 \\ Institut für Theoretische Physik, Technische Universität Wien, \\ Wiedner Hauptstrasse 8-10, A-1040 Vienna, Austria
}

(Received 7 September 2021; accepted 10 October 2021; published 12 November 2021)

\begin{abstract}
We extend our previous calculations of the hadronic light-by-light scattering contribution to the muon anomalous magnetic moment in holographic QCD to models with finite quark masses and a tower of massive pions. Analyzing the role of the latter in the Wess-Zumino-Witten action, we show that the Melnikov-Vainshtein short-distance constraint is satisfied solely by the summation of contributions from the infinite tower of axial-vector meson contributions. There is also an enhancement of the asymptotic behavior of pseudoscalar contributions when their infinite tower of excitations is summed, but this leads only to subleading contributions for the short-distance constraints on light-by-light scattering. We also refine our numerical evaluations, particularly in the pion and $a_{1}$ sector, which corroborates our previous findings of contributions from axial-vector mesons that are significantly larger than those adopted for the effects of axials and short-distance constraints in the recent white paper on the Standard Model prediction for $(g-2)_{\mu}$.

DOI: 10.1103/PhysRevD.104.094017
\end{abstract}

\section{INTRODUCTION}

Recently, the Muon $g-2$ Collaboration at Fermilab [1] has confirmed the long-standing discrepancy between the E821/BNL measurement [2] of the anomalous magnetic moment of the muon [3] and the Standard Model prediction, which according to the white paper (WP) of the Muon $g-2$ Theory Initiative [4] is below the combined experimental value by an amount of $\Delta a_{\mu}=\Delta(g-2)_{\mu} / 2=$ $251(59) \times 10^{-11}$.

While QED [5] and electroweak effects [6,7] appear to be fully under control, the theoretical uncertainty is dominated by hadronic effects [8-28]. The largest contribution by far is the hadronic vacuum polarization, where a recent lattice calculation [29] has challenged the WP result, albeit by producing tensions in other quantities [30-32] - an issue which further lattice calculations as well as new experimental results on hadronic cross sections should resolve in the near future. The second-largest uncertainty comes from hadronic light-by-light scattering (HLBL) [26], which also needs improvements in view of the expected reductions of experimental errors by the ongoing experiment at Fermilab and a new one in preparation at J-PARC [33].

Published by the American Physical Society under the terms of the Creative Commons Attribution 4.0 International license. Further distribution of this work must maintain attribution to the author(s) and the published article's title, journal citation, and DOI. Funded by SCOAP ${ }^{3}$.
At the low energies relevant for the evaluation of $a_{\mu}$, the HLBL amplitude is dominated by the two-photon coupling of the pion, where the transition form factors (TFFs) needed to evaluate the pion pole contribution have been computed with satisfying accuracy in data-driven approaches [14,21] which are also in agreement with results from lattice QCD [22]. Among the many additional low-energy contributions, those contributed by axial-vector mesons are currently the ones involving the largest uncertainties due to insufficient experimental data. Axial-vector mesons also play a crucial role in connecting the HLBL amplitude at low energies to short-distance constraints (SDCs), in particular the one derived by Melnikov and Vainshtein (MV) [8] using the nonrenormalization theorem for the vector-vector-axial-vector (VVA) correlator and the axial anomaly, as has been clarified by holographic models of QCD (hQCD) [34,35], which are the first hadronic models to implement fully the constraints [36,37] imposed by the axial anomaly in the chiral limit.

Away from the chiral limit, excited pseudoscalars also couple to the axial-vector current, and thus to the axial anomaly. In Refs. [24,25], a Regge model for excited pseudoscalars has been constructed to satisfy the longitudinal SDCs (LSDCs) and to estimate the corresponding contributions to $a_{\mu}$. In this paper, we employ the simplest hard-wall AdS/QCD model that is capable of satisfying at the same time the leading-order (LO) perturbative QCD (pQCD) constraints on the vector correlator and the lowenergy parameters provided by the pion decay constant $f_{\pi}$ 
and the mass of the $\rho$ meson, introduced in Refs. [38,39] and called HW1 in Refs. [34,40,41], using here its generic form with finite quark masses (albeit in the simplest version of uniform quark masses). This allows us to study the role of massive pions in the axial anomaly and in the longitudinal SDCs. We find that in hQCD, there is in fact a certain enhancement of the asymptotic behavior when summing the infinite tower of pseudoscalars compared to the behavior of individual contributions, but within the allowed range of parameters of hQCD this is insufficient to let pseudoscalars contribute to the leading terms of the longitudinal SDCs; the latter are determined by the infinite tower of axial-vector mesons alone, in agreement with the expectation expressed in Ref. [35].

Moreover, we evaluate and assess a set of massive hardwall AdS/QCD models with regard to their predictions for the pseudoscalar and axial-vector TFFs and the resulting contributions to $a_{\mu}$, also allowing for adjustments at high energies to account for next-to-leading-order (NLO) pQCD corrections. The results for the single- and double-virtual pion TFFs agree perfectly with those of the data-driven dispersive results of Ref. [22], in particular when a $10 \%$ reduction of the asymptotic limit is applied. The shape of the axial-vector TFF is consistent with L3 results for the $f_{1}(1285)$ axial-vector meson [42]; the magnitude is above experimental values, but it becomes compatible after such reductions. While hQCD is clearly only a toy model for real QCD, this success after a fit of a minimal set of low-energy parameters seems to make it also interesting as a phenomenological model (which may be further improved by using a modified background geometry and other refinements [43-45]).

Numerically, the axial-vector contributions are close to our previous results for the HW1 model in the chiral limit (they even tend to increase with finite quark masses), and they are thus significantly larger than estimated in the WP [4]. Interestingly enough, a new complete lattice calculation which claims comparable errors [28] has obtained somewhat larger values of $a_{\mu}^{\mathrm{HLBL}}$ than the WP [4], which could also be indicative of larger contributions from the axial-vector meson sector.

The contributions from the excited pseudoscalars are numerically much smaller than those from axial-vector mesons. They are present also in the chiral HW1 model, where they decouple from the axial-vector current and the axial anomaly, but not from low-energy photons. However, the first excited pion mode has a two-photon coupling that is significantly above the experimental constraint deduced in Ref. [25]. Nevertheless, the contribution of the first few excited modes together is smaller than those of the Regge model of Refs. [24,25], which is compatible with known experimental constraints. This model was, however, not meant primarily as a phenomenological model for estimating the contributions of excited pseudoscalars, but rather as a model for estimating the effects of the longitudinal SDCs, which according to the hQCD models are instead provided by the axial-vector mesons. As far as these effects are concerned, our present results are not far above the estimates obtained in Refs. [24,25,46,47]; all are significantly below the estimates obtained with the so-called MV model [8], where the ground-state pion contribution is modified.

This paper is organized as follows: In Sec. II, we review hQCD with an anti-de Sitter (AdS) background that is cut off by a hard wall, and where in addition to flavor gauge fields, a bifundamental scalar bulk field encodes the chiral condensates with or without finite quark masses. In addition to the boundary conditions considered originally in Refs. [38,39] where vector and axial-vector gauge fields are treated equally (HW1), we also consider the other set of admissible boundary conditions that appear in models without a bifundamental scalar, the Hirn-Sanz (HW2) model [48] and the top-down hQCD model of Sakai and Sugimoto [49,50]. (This modification of the HW1 model will be referred to as HW3.) As has been pointed out in Ref. [51], this has the advantage of removing infrared boundary terms in the Chern-Simons action that in the HW1 model need to be subtracted by hand [52]. Additionally, we consider the generalization of different scaling dimensions for quark masses and chiral condensates proposed in Ref. [51], which permits us to fit the mass of the first excited pion or the lowest axial-vector meson. In Sec. III, we analyze the consequences of the axial anomaly in the HW models - in particular, the role of excited pseudoscalars-as encoded by the bulk Chern-Simons action for the five-dimensional gauge fields. In Sec. IV, we study SDCs on TFFs and the HLBL scattering amplitude, showing analytically that the MV-SDC is always saturated by axial-vector mesons, while the symmetric longitudinal SDC is fulfilled to $81 \%$. Within the bounds of allowed scaling dimensions for the bulk bifundamental scalar, the infinite tower of excited pseudoscalar cannot change this. Finally, in Sec. V, we evaluate the set of massive HW models numerically, comparing the resulting masses, decay constants, and TFFs with empirical data, before calculating the corresponding contributions to $a_{\mu}$.

\section{HARD-WALL ADS/QCD MODELS WITH FINITE QUARK MASSES}

In the hard-wall AdS/QCD models of Refs. [38,39,48], the background geometry is chosen as pure anti-de Sitter with the metric

$$
d s^{2}=z^{-2}\left(\eta_{\mu \nu} d x^{\mu} d x^{\nu}-d z^{2}\right),
$$

with a conformal boundary at $z=0$. Confinement is implemented by a cutoff at some finite value of the radial coordinate $z_{0}$, where boundary conditions for the fivedimensional fields are imposed. 
Left and right chiral quark currents are dual to fivedimensional flavor gauge fields, whose action is given by a Yang-Mills part $^{1}$

$$
\begin{aligned}
S_{\mathrm{YM}}= & -\frac{1}{4 g_{5}^{2}} \int d^{4} x \int_{0}^{z_{0}} d z \sqrt{-g} g^{P R} g^{Q S} \\
& \times \operatorname{tr}\left(\mathcal{F}_{P Q}^{\mathrm{L}} \mathcal{F}_{R S}^{\mathrm{L}}+\mathcal{F}_{P Q}^{\mathrm{R}} \mathcal{F}_{R S}^{\mathrm{R}}\right),
\end{aligned}
$$

where $P, Q, R, S=0, \ldots, 3, z$ and $\mathcal{F}_{M N}=\partial_{M} \mathcal{B}_{N}-\partial_{N} \mathcal{B}_{M}-$ $i\left[\mathcal{B}_{M}, \mathcal{B}_{N}\right]$, and a Chern-Simons action $S_{\mathrm{CS}}=S_{\mathrm{CS}}^{\mathrm{L}}-S_{\mathrm{CS}}^{\mathrm{R}}$, with (in differential form notation)

$$
S_{\mathrm{CS}}^{\mathrm{L}, \mathrm{R}}=\frac{N_{c}}{24 \pi^{2}} \int \operatorname{tr}\left(\mathcal{B} \mathcal{F}^{2}-\frac{i}{2} \mathcal{B}^{3} \mathcal{F}-\frac{1}{10} \mathcal{B}^{5}\right)^{\mathrm{L}, \mathrm{R}},
$$

up to a potential subtraction of boundary terms at $z_{0}$ to be discussed below.

As a dual field for the scalar quark bilinear operator, a bifundamental bulk scalar [38,39] $X$, parametrized as [53]

$$
X=e^{i \pi^{a}(x, z) t^{a}}\left[\frac{1}{2} v(z)\right] e^{i \pi^{a}(x, z) t^{a}},
$$

is introduced, with the action

$$
S_{X}=\int d^{4} x \int_{0}^{z_{0}} d z \sqrt{-g} \operatorname{tr}\left(|D X|^{2}-M_{X}^{2}|X|^{2}\right),
$$

where $D_{M} X=\partial_{M} X-i \mathcal{B}_{M}^{\mathrm{L}} X+i X \mathcal{B}_{M}^{\mathrm{R}}$. The five-dimensional mass term is determined by the scaling dimension of the chiral-symmetry-breaking order parameter $\bar{q}_{L} q_{R}$ of the boundary theory. With $M_{X}^{2}=-3$, one obtains vacuum solutions $v(z)=M_{q} z+\Sigma z^{3}$, where $M_{q}$ and $\Sigma$ are model parameters related to the quark mass matrix and the quark condensate. ${ }^{2}$ Following Ref. [51], we shall also consider generalizations away from $M_{X}^{2}=-3$, where

$$
v(z)=M_{q} z_{0}\left(z / z_{0}\right)^{\Delta^{-}}+\Sigma z_{0}^{3}\left(z / z_{0}\right)^{\Delta^{+}}
$$

with

$$
\Delta^{ \pm}=2 \pm \sqrt{4+M_{X}^{2}} \equiv 2 \pm \alpha .
$$

In the original hard-wall model of Refs. [38,39] (HW1), chiral-symmetry-preserving boundary conditions are employed at the infrared cutoff, $\mathcal{F}_{z \mu}^{\mathrm{L}, \mathrm{R}}\left(z_{0}\right)=0$, whereas in the model of Hirn and Sanz [48] (called HW2 in Refs. $[34,40,41])$, chiral symmetry is broken through $\left(\mathcal{B}_{\mu}^{\mathrm{L}}-\mathcal{B}_{\mu}^{\mathrm{R}}\right)\left(z_{0}\right)=0$ and $\left(\mathcal{F}_{z \mu}^{\mathrm{L}}+\mathcal{F}_{z \mu}^{\mathrm{R}}\right)\left(z_{0}\right)=0$ without the introduction of a bifundamental scalar. These latter

\footnotetext{
${ }^{1}$ Note that in our conventions, the metric $g_{M N}$ carries a dimension of inverse length squared; $g_{5}$ is therefore dimensionless.

${ }^{2}$ According to Refs. [39,53-55], these need to be rescaled by $C=\sqrt{N_{c}} /(2 \pi)$ before being interpreted as quark masses and condensates: $m_{q}=M_{q} / C$ and $\langle q \bar{q}\rangle=C \Sigma$.
}

conditions arise naturally in the top-down holographic chiral model of Sakai and Sugimoto $[49,50]$ with flavor gauge fields residing on flavor branes separated by an extra dimension at the boundary but connecting in the bulk. In Ref. [51], it was proposed to use such symmetry-breaking boundary conditions also in the presence of symmetry breaking by a bifundamental scalar, because it avoids infrared boundary contributions from the CS action. This variant of the HW1 model will be termed HW3 in the following.

In both the HW1 and the HW3 models, chiral symmetry can be broken additionally by finite quark masses. In the present paper, we shall only consider the flavor-symmetric case, where both $M_{q}$ and $\Sigma$ are proportional to the unit matrix. For both models, we will also consider generalizations away from $M_{X}^{2}=-3$, which, as Ref. [51] has found, allows the HW3 model to fit simultaneously the masses of the lightest and the first excited pion. As shown in Appendix A, all these models lead to a Gell-Mann-Oakes-Renner (GOR) relation in the limit of small quark masses- to wit,

$$
f_{\pi}^{2} m_{\pi}^{2}=2 \alpha M_{q} \Sigma \quad \text { for } M_{q} \ll \Sigma z_{0}^{2} \propto \Sigma / m_{\rho}^{2}
$$

where $\alpha=1$ for the standard choice $M_{X}^{2}=-3$, and $0<\alpha<2$ for the admissible generalizations [51] $-4<M_{X}^{2}<0$.

\section{A. Vector sector}

As long as flavor symmetry is in place, vector mesons, which appear in the mode expansion of $V_{\mu}=\left(\mathcal{B}_{\mu}^{L}+\mathcal{B}_{\mu}^{R}\right) / 2$, have (for all hard-wall models considered here) quark-mass independent holographic wave functions given by

$$
\partial_{z}\left[\frac{1}{z} \partial_{z} \psi_{n}(z)\right]+\frac{1}{z} M_{n}^{2} \psi_{n}(z)=0
$$

with boundary conditions $\psi_{n}(0)=\psi_{n}^{\prime}\left(z_{0}\right)=0$. This is solved by $\psi_{n}(z) \propto z J_{1}\left(M_{n} z\right)$, with $M_{n}$ determined by the zeros of the Bessel function $J_{0}$, denoted by $\gamma_{0, n}$. With normalization $\int_{0}^{z_{0}} d z z^{-1} \psi_{n}(z)^{2}=1$, we have

$$
\psi_{n}(z)=\sqrt{2} \frac{z J_{1}\left(\gamma_{0, n} z / z_{0}\right)}{z_{0} J_{1}\left(\gamma_{0, n}\right)}
$$

and canonically normalized vector meson fields $v_{\mu}(x)$ in the mode expansion $V_{\mu}^{a}=g_{5} \sum_{n=1}^{\infty} v_{\mu}^{a}(x) \psi_{n}(z)$.

Identifying $M_{1}=\gamma_{0,1} / z_{0}=m_{\rho}=775 \mathrm{MeV}$, we obtain

$$
z_{0}=\gamma_{0,1} / m_{\rho}=3.103 \mathrm{GeV}^{-1}
$$

The vector bulk-to-boundary propagator is obtained by replacing $M_{n}^{2}$ with $q^{2}=-Q^{2}$ and the boundary conditions with $\mathcal{J}(Q, 0)=1$ and $\partial_{z} \mathcal{J}\left(Q, z_{0}\right)=0$. This gives 


$$
\begin{aligned}
\mathcal{J}(Q, z) & =Q z\left[K_{1}(Q z)+\frac{K_{0}\left(Q z_{0}\right)}{I_{0}\left(Q z_{0}\right)} I_{1}(Q z)\right] \\
& =\sum_{n=1}^{\infty} \frac{g_{5} F_{n}^{V} \psi_{n}(z)}{Q^{2}+M_{n}^{2}}
\end{aligned}
$$

where $F_{n}^{V}=\left|\psi_{n}^{\prime}(\epsilon) /\left(g_{5} \epsilon\right)\right|$ is the decay constant of $v_{n}(x)$,

$$
\left\langle 0\left|J_{\mu}^{a}(0)\right| v_{n}^{b}(q, \lambda)\right\rangle=F_{n}^{V} \varepsilon_{\mu}(q, \lambda) \delta^{a b} .
$$

Requiring that the vector current two-point function match the leading-order pQCD result [38]

$$
\Pi_{V}\left(Q^{2}\right)=-\left.\frac{1}{g_{5}^{2} Q^{2}}\left(\frac{1}{z} \partial_{z} \mathcal{J}(Q, z)\right)\right|_{z \rightarrow 0}=-\frac{N_{c}}{24 \pi^{2}} \ln Q^{2}
$$

determines $g_{5}^{2}=12 \pi^{2} / N_{c}$.

The simpler Hirn-Sanz (HW2) model [48], which does not include the scalar $X$, cannot set $g_{5}^{2}$ to the value required by $\mathrm{pQCD}$, when the mass of the rho meson and the pion decay constant are matched to experiment (as we shall do throughout).

\section{B. Axial sector}

Following the notation of Ref. [53], the five-dimensional Lagrangian contains the following quadratic terms for the axial gauge field $A_{M}=\left(\mathcal{B}_{M}^{L}-\mathcal{B}_{M}^{R}\right) / 2$ and the pseudoscalar $\pi$ :

$$
\begin{aligned}
\mathcal{L}_{\text {axial }} & =-\frac{1}{4 g_{5}^{2} z}\left(\partial_{M} A_{N}-\partial_{N} A_{M}\right)^{2}+\frac{\beta(z)}{2 g_{5}^{2} z}\left(\partial_{M} \pi-A_{M}\right)^{2}, \\
\beta(z) & =g_{5}^{2} v^{2} / z^{2}
\end{aligned}
$$

where five-dimensional indices are implicitly contracted with a five-dimensional Minkowski metric $\eta^{M N}$ (mostly minus).

Axial-vector mesons are contained in $A_{\mu}^{\perp}=g_{5}$ $\sum_{n=1}^{\infty} a_{\mu}^{(n)}(x) \psi_{n}^{A}(z)$, with the holographic wave functions subject to

$$
\partial_{z}\left[\frac{1}{z} \partial_{z} \psi_{n}^{A}(z)\right]+\frac{1}{z}\left[M_{A, n}^{2}-\beta(z)\right] \psi_{n}^{A}(z)=0,
$$

which is different from Eq. (9) when $v \neq 0$, and so gives a different mass spectrum even when vector and axial-vector fields have identical boundary conditions, as is the case in the HW1 model. In the HW3 model, chiral symmetry is additionally broken by the Dirichlet boundary condition $\psi_{n}^{A}\left(z_{0}\right)_{\mathrm{HW} 3}=0$.

In the holographic gauge $A_{z}=0$, the pseudoscalar $\pi$ mixes with the longitudinal part of the axial-vector gauge field denoted by $A_{\mu}^{\|}=\partial_{\mu} \phi$,

$$
\begin{aligned}
\mathcal{L}_{\text {axial }} \supset & \frac{1}{2 g_{5}^{2} z}\left(\partial_{\mu} \partial_{z} \phi\right)^{2}+\frac{1}{2 g_{5}^{2} z} \beta(z)\left(\partial_{\mu} \pi-\partial_{\mu} \phi\right)^{2} \\
& -\frac{1}{2 g_{5}^{2} z} \beta(z)\left(\partial_{z} \pi\right)^{2} .
\end{aligned}
$$

Alternatively, one may decouple $A_{\mu}$ from $A_{z}$ and $\pi$ by adding the $R_{\xi}$ gauge-fixing term [51]

$$
\mathcal{L}_{R_{\xi}}=-\frac{1}{2 \xi z g_{5}^{2}}\left[\partial_{\mu} A^{\mu}-\xi z \partial_{z}\left(\frac{1}{z} A_{z}\right)+\xi \beta \pi\right]^{2} .
$$

Taking the $\xi \rightarrow \infty$ limit imposes the unitary gauge, which fixes

$$
z \partial_{z}\left(\frac{1}{z} A_{z}^{\mathrm{U}}\right)=\beta \pi^{\mathrm{U}}
$$

In the unitary gauge, $A_{\mu}^{\mathrm{U}}$ is a simple Proca field, and the relation to the fields in the holographic gauge (the latter written without label) are $A_{M}^{\mathrm{U}}=A_{M}-\partial_{M} \phi, \pi^{\mathrm{U}}=\pi-\phi$, with $A_{\mu}^{\| \mathrm{U}}=0$ and $A_{z}^{\mathrm{U}}=-\partial_{z} \phi$.

The equations of motion for $\phi$ and $\pi$ read

$$
\begin{gathered}
\partial_{z}\left(\frac{1}{z} \partial_{z} \phi\right)+\frac{\beta(z)}{z}(\pi-\phi)=0, \\
\partial_{z}\left(\frac{\beta(z)}{z} \partial_{z} \pi\right)+\frac{\beta(z)}{z} q^{2}(\pi-\phi)=0 .
\end{gathered}
$$

Defining $y=\partial_{z} \phi / z$, one can combine these coupled equations into a single second-order differential equation $[53,56]$ :

$\left(L+q^{2}\right) y:=\frac{\beta(z)}{z} \partial_{z}\left(\frac{z}{\beta(z)} \partial_{z} y\right)-\beta(z) y+q^{2} y=0$.

The modes for $\pi$ follow from Eqs. (20) and (21), which imply

$$
\partial_{z} y=\frac{\beta(z)}{z}(\phi-\pi)
$$

and

$$
\partial_{z} \pi=\frac{q^{2}}{\beta(z)} z y .
$$

The boundary conditions at $z=\epsilon \rightarrow 0$ are $\phi_{S}(q, \epsilon)=0$, $\pi_{S}(q, \epsilon)=-1$ for the non-normalizable profile functions, and $\phi_{n}(\epsilon)=0, \pi_{n}(\epsilon)=0$ for the normalizable modes. This implies

$$
\left.\frac{z}{\beta(z)} \partial_{z} y_{S}(q, z)\right|_{z=\epsilon}=1,\left.\quad \frac{z}{\beta(z)} \partial_{z} y_{n}(z)\right|_{z=\epsilon}=0 .
$$


At the infrared boundary $z=z_{0}$, there are two possible choices which eliminate the boundary term in the axial part of the action

$\frac{1}{2 g_{5}^{2} z_{0}}\left(q^{2} \phi \partial_{z} \phi-\beta \pi \partial_{z} \pi\right)_{z=z_{0}}=\frac{q^{2}}{2 g_{5}^{2}} y\left(z_{0}\right)\left[\phi\left(z_{0}\right)-\pi\left(z_{0}\right)\right]$.

In the HW1 model, one has Neumann boundary conditions at $z=z_{0}$ for all gauge fields, and therefore on $\phi$; Eq. (26) is then canceled by requiring $y\left(z_{0}\right)=0$. In the HW3 model, where the axial gauge field has Dirichlet boundary conditions, one chooses instead $\left.\partial_{z} y\right|_{z=z_{0}}=0$, which through Eq. (23) implies $\left[\phi\left(z_{0}\right)-\pi\left(z_{0}\right)\right]=0$. The HW2/HW3 boundary condition $A_{\mu}\left(z_{0}\right)=0$ thus only applies to $A_{\mu}^{\perp}\left(z_{0}\right)=0$ when the holographic gauge $A_{z}=0$ is employed, where $A_{\mu}^{\|}=\partial_{\mu} \phi$; in the unitary gauge, the latter is transferred to $A_{z}^{\mathrm{U}}=-\partial_{z} \phi=-z y$.

The normalizable modes (the first few of which are shown in Fig. 1) are normalized by

$$
\int_{\epsilon}^{z_{0}} d z \frac{z}{\beta(z)} y_{n} y_{m}=\int_{\epsilon}^{z_{0}} d z \frac{1}{z \beta(z)} \partial_{z} \phi_{n} \partial_{z} \phi_{m}=\frac{\delta_{m n}}{m_{n}^{2}}
$$

so that canonically normalized pion fields $\Pi_{n}(x)$ appear in a mode expansion according to

$\phi=g_{5} \sum_{n=1}^{\infty} \Pi_{n}(x) \phi_{n}(z), \quad \pi=g_{5} \sum_{n=1}^{\infty} \Pi_{n}(x) \pi_{n}(z)$.

In unitary gauge, one has

$$
A_{z}^{\mathrm{U}}=-\partial_{z} \phi=-g_{5} \sum_{n=1}^{\infty} \Pi_{n}(x) z y_{n}(z) .
$$

Normalizable and non-normalizable $y$ modes are related by the sum rule [53]

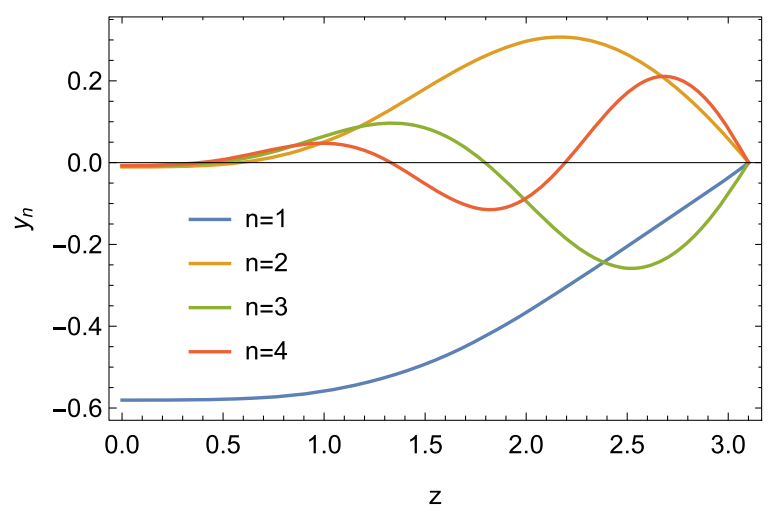

$y_{S}(q, z)=\sum_{n} \frac{m_{n}^{2} y_{n}(\epsilon) y_{n}(z)}{q^{2}-m_{n}^{2}}=-g_{5} \sum_{n=1}^{\infty} \frac{f_{\pi_{n}} m_{n}^{2} y_{n}(z)}{q^{2}-m_{n}^{2}}$,

where $m_{n}$ 's are the masses of the tower of pions and $f_{\pi_{n}}=-y_{n}(\epsilon) / g_{5}$ are their decay constants,

$$
\left\langle 0\left|J_{\| \mu}^{A, a}(0)\right| \pi_{n}^{b}(q)\right\rangle=i f_{\pi_{n}} q_{\mu} \delta^{a b} .
$$

For later use, we also define the Green's function

$$
\frac{z}{\beta(z)}\left(L+q^{2}\right) G\left(z, z^{\prime} ; q\right)=-\delta\left(z-z^{\prime}\right)
$$

with boundary conditions as imposed on the mode functions $y_{n}$, so that

$$
G\left(z, z^{\prime} ; q\right)=-\sum_{n} \frac{m_{n}^{2} y_{n}(z) y_{n}\left(z^{\prime}\right)}{q^{2}-m_{n}^{2}}
$$

In the chiral limit, the infinite tower of massive pions continues to be present, but their decay constants vanish: $f_{\pi_{n}} \rightarrow 0$ for $n \geq 2$ as $M_{q} \rightarrow 0$. The mode $n=1$ becomes massless with $y_{S} \rightarrow g_{5} f_{\pi} y_{1}, f_{\pi}=f_{\pi_{1}}$. (See Appendix A for more details.)

\section{AXIAL ANOMALY AND MASSIVE PIONS}

The Chern-Simons action [Eq. (3)] implements the axial anomaly and the associated $V V A$ coupling $[52,57]$. After integration over the holographic direction, one obtains a Wess-Zumino-Witten action for the mesons. This contains vertices involving photons, which are described by $V_{\mu}(x, z)=A_{\mu}^{\text {e.m. }}(x) \mathcal{Q} \mathcal{J}(z)$, with $\mathcal{Q}$ denoting the charge matrix of quarks, and the fields encoded by $A_{M}$, namely the infinite towers of pions and axial-vector mesons.

In the chiral limit, where all massive pions decouple from the axial-vector current through vanishing decay constants, the resulting pion TFF has been analyzed in various holographic QCD models in Refs. [58,59] and also

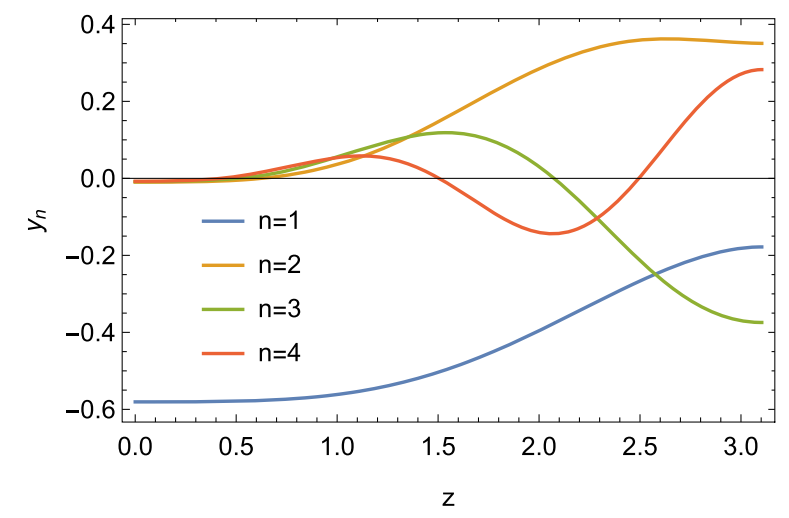

FIG. 1. The first four pion-mode functions $y_{n}(z)$ in the HW1m model and in the HW3m version (precise definitions in Sec. V), with $z$ in units of inverse $\mathrm{GeV}$; the $y_{n}$ values have units of $\mathrm{GeV}$, with $y_{n}(0)=-g_{5} f_{\pi_{n}}$. 
in Refs. [40,41], where the HLBL contribution to the anomalous magnetic moment of the muon was studied. This was also done in Ref. [60] for the ground-state pseudoGoldstone bosons in a version of the HW1 model with finite quark masses.

The contribution of the infinite tower of axial-vector mesons, which is crucial for satisfying the MelnikovVainshtein short-distance constraint, has been worked out in Refs. [34,35] for the chiral version of the HW1 model and the inherently chiral Hirn-Sanz (HW2) model. We refer to these latter references for details on the axialvector contributions, which, as we shall see, do not change qualitatively away from the chiral limit, concentrating here on the generalizations necessary to include the contributions of massive pions in the HW1 and HW3 models.

In the holographic gauge $A_{z}=0$, the tower of pions contributes to the Chern-Simons action through $A_{\mu}^{\|}=\partial_{\mu} \phi$, whereas in the unitary gauge it appears in $A_{z}^{\mathrm{U}}=\partial_{z} \phi$. In the latter case, the anomalous interactions of the pions are described by

$S_{(\pi)}^{\mathrm{anom}}=\frac{N_{c}}{4 \pi^{2}} \epsilon^{\mu \nu \rho \sigma} \operatorname{tr} \int d^{4} x \int_{0}^{z_{0}} d z A_{z}^{\mathrm{U}} \partial_{\mu} V_{\nu} \partial_{\rho} V_{\sigma}-B$,

where $B$ is an infrared subtraction term required by the HW1 model and introduced in Ref. [52], but which disappears in the HW2 and HW3 models due to their different boundary conditions at $z=z_{0}$.

With Eq. (29), this yields the following pion TFFs [for which $\left.\operatorname{tr}\left(t^{3} \mathcal{Q}^{2}\right)=1 / 6\right]$ :

$$
F_{\pi_{n} \gamma^{*} \gamma^{*}}\left(Q_{1}^{2}, Q_{2}^{2}\right)=\frac{N_{c}}{12 \pi^{2}} g_{5} K_{n}\left(Q_{1}^{2}, Q_{2}^{2}\right),
$$

with

$$
\begin{aligned}
K_{n}\left(Q_{1}^{2}, Q_{2}^{2}\right)= & -\int_{0}^{z_{0}} d z \mathcal{J}\left(Q_{1}, z\right) \mathcal{J}\left(Q_{2}, z\right) z y_{n}(z) \\
& +\mathcal{J}\left(Q_{1}, z_{0}\right) \mathcal{J}\left(Q_{2}, z_{0}\right)\left(\frac{z}{\beta} \partial_{z} y_{n}(z)\right)_{z \rightarrow z 0},
\end{aligned}
$$

where the last term vanishes automatically in the HW3 model.

This can also be written in terms of $\phi$ and $\pi$ modes,

$$
\begin{aligned}
K_{n}\left(Q_{1}^{2}, Q_{2}^{2}\right)= & -\int_{0}^{z_{0}} d z \mathcal{J}\left(Q_{1}, z\right) \mathcal{J}\left(Q_{2}, z\right) \partial_{z} \phi_{n}(z) \\
& +\mathcal{J}\left(Q_{1}, z_{0}\right) \mathcal{J}\left(Q_{2}, z_{0}\right)\left[\phi_{n}\left(z_{0}\right)-\pi_{n}\left(z_{0}\right)\right],
\end{aligned}
$$

which implies (equally in the HW1 and HW3 models)

$$
K_{n}(0,0)=-\pi_{n}\left(z_{0}\right),
$$

since $\mathcal{J}(0, z) \equiv 1$. In the chiral limit, one has a constant $\pi_{1}(z) \equiv-1 /\left(g_{5} f_{\pi_{1}}\right)$ as the sole contribution, yielding $F_{\pi_{1} \gamma \gamma} \equiv F_{\pi_{1} \gamma^{*} \gamma^{*}}(0,0)=N_{c} /\left(12 \pi^{2} f_{\pi_{1}}\right)$.

We note parenthetically that the above subtraction term agrees with the one introduced in Ref. [52], but the bulk term therein involves $\partial_{z}(\phi-\pi)$, which is correct only for the $n=1$ mode in the chiral limit where $\pi_{1}(z)$ becomes a constant. Away from the chiral limit, the expression proposed in Ref. [52] would in fact give $K_{n}(0,0)=0$ for all $n$, because the boundary conditions on the normalizable modes of $\phi$ and $\pi$ are such that they vanish at $z=0$.

With nonzero quark masses, $K_{n}(0,0)$ can only be given numerically. However, the sum rule [Eq. (30)] implies that $y_{S}(0, z)=g_{5} \sum_{n=1}^{\infty} f_{\pi_{n}} y_{n}(z)$, and consequently

$$
g_{5} \sum_{n=1}^{\infty} f_{\pi_{n}} K_{n}(0,0)=1 \text {, }
$$

or

$$
\sum_{n=1}^{\infty} f_{\pi_{n}} F_{\pi_{n} \gamma \gamma}=\frac{N_{c}}{12 \pi^{2}}
$$

showing that all massive pions then contribute to the anomaly. In Fig. 2, this is illustrated with the HW1m model (specified in Sec. V).

However, also in the chiral limit, where $f_{\pi_{n}} \rightarrow 0$ for $n>1$ so that the massive pions decouple from the axial-vector current and the axial anomaly, $K_{n}(0,0)$ and $F_{\pi_{n} \gamma \gamma}$ remain nonzero.

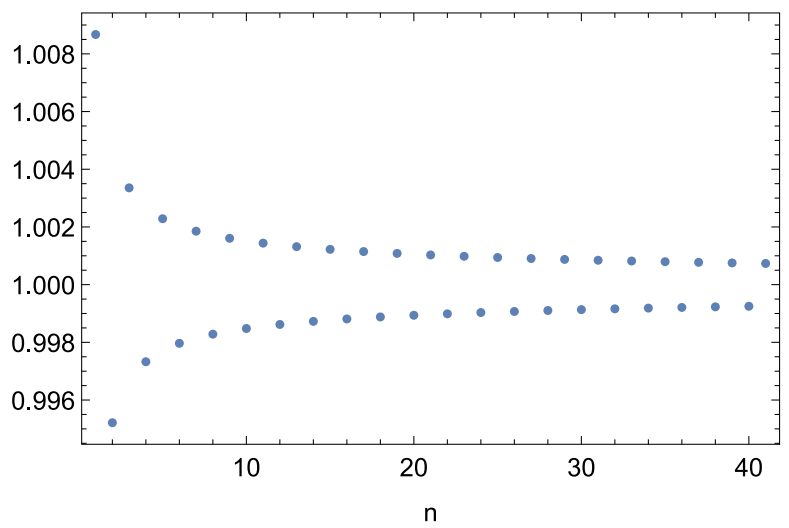

FIG. 2. Partial sums of the sum relation in Eq. (39) as a function of the number of summed modes for the HW1m model. 


\section{SHORT-DISTANCE CONSTRAINTS}

\section{A. Form factors}

Remarkably, the short-distance constraints of pQCD on meson TFFs [61-63], including their dependence on the ratio $w=\left(Q_{1}^{2}-Q_{2}^{2}\right) /\left(Q_{1}^{2}+Q_{2}^{2}\right)$, are reproduced exactly by the HW models in the limit $Q_{i}^{2} \rightarrow \infty$. With $Q^{2}=\frac{1}{2}\left(Q_{1}^{2}+Q_{2}^{2}\right) \rightarrow \infty$, one finds

$F_{\pi_{n} \gamma^{*} \gamma^{*}}\left(Q_{1}^{2}, Q_{2}^{2}\right) \rightarrow \frac{g_{5}^{2} N_{c}}{12 \pi^{2}} \frac{2 f_{\pi_{n}}}{Q^{2}}\left[\frac{1}{w^{2}}-\frac{1-w^{2}}{2 w^{3}} \ln \frac{1+w}{1-w}\right]$,

generalizing the chiral result [58] to all massive pions. Thus, with $g_{5}$ fixed by Eq. (14), all satisfy the Brodsky-Lepage limit [61] with their respective decay constants, $\lim _{Q^{2} \rightarrow \infty} Q^{2} F_{\pi_{n} \gamma^{*} \gamma^{*}}\left(Q^{2}, 0\right)=2 f_{\pi_{n}}$, and likewise the symmetric pQCD limit [64], $\lim _{Q^{2} \rightarrow \infty} Q^{2} F_{\pi_{n} \gamma^{*} \gamma^{*}}$ $\left(Q^{2}, Q^{2}\right)=2 f_{\pi_{n}} / 3$.

The amplitude for axial-vector mesons $a_{\mu}^{(n)}$ decaying into two virtual photons following from the Chern-Simons action has the form $[34,35]$

$$
\begin{aligned}
\mathcal{M}^{a}= & i \frac{N_{c}}{4 \pi^{2}} \operatorname{tr}\left(\mathcal{Q}^{2} t^{a}\right) \epsilon_{(1)}^{\mu} \epsilon_{(2)}^{\nu} \epsilon_{A}^{* \rho} \epsilon_{\mu \nu \rho \sigma} \\
& \times\left[q_{(2)}^{\sigma} Q_{1}^{2} A_{n}\left(Q_{1}^{2}, Q_{2}^{2}\right)-q_{(1)}^{\sigma} Q_{2}^{2} A_{n}\left(Q_{2}^{2}, Q_{1}^{2}\right)\right],
\end{aligned}
$$

where

$$
A_{n}\left(Q_{1}^{2}, Q_{2}^{2}\right)=\frac{2 g_{5}}{Q_{1}^{2}} \int_{0}^{z_{0}} d z\left[\frac{d}{d z} \mathcal{J}\left(Q_{1}, z\right)\right] \mathcal{J}\left(Q_{2}, z\right) \psi_{n}^{A}(z),
$$

which has a finite limit when $Q_{1}^{2} \rightarrow 0$. Hence, Eq. (42) vanishes when both photons are real, in accordance with the Landau-Yang theorem $[65,66]$.

The asymptotic behavior of Eq. (43) reads [34]

$$
\begin{aligned}
A_{n}\left(Q_{1}^{2}, Q_{2}^{2}\right) \rightarrow & \frac{g_{5}^{2} F_{n}^{A}}{Q^{4}} \frac{1}{w^{4}}[w(3-2 w) \\
& \left.+\frac{1}{2}(w+3)(1-w) \ln \frac{1-w}{1+w}\right],
\end{aligned}
$$

which also agrees with the $\mathrm{pQCD}$ behavior derived recently in Ref. [63].

Compared to the most general expression possible for the axial-vector amplitude $\mathcal{M}\left(a \rightarrow \gamma^{*} \gamma^{*}\right)$ [67-69], the holographic result in Eq. (42) has one asymmetric structure function [denoted $C\left(Q_{1}^{2}, Q_{2}^{2}\right)$ in Ref. [68] ] set to zero; see Ref. [69] for a compilation of the available phenomenological information.

\section{B. Longitudinal short-distance constraint on HLBL amplitude}

$$
\text { 1. } Q_{1}^{2}=Q_{2}^{2} \gg Q_{3}^{2} \gg m_{\rho}^{2}
$$

In the Bardeen-Tung-Tarrach basis of the HLBL fourpoint function [70], the longitudinal short-distance constraint of Melnikov and Vainshtein [8] in the region $Q_{1}^{2} \sim Q_{2}^{2} \gg Q_{3}^{2} \gg m_{\rho}^{2}$ and $Q_{4}=0$, which is governed by the chiral anomaly and protected by its nonrenormalization theorem, reads [24,25]

$$
\lim _{Q_{3} \rightarrow \infty} \lim _{Q \rightarrow \infty} Q^{2} Q_{3}^{2} \bar{\Pi}_{1}\left(Q, Q, Q_{3}\right)=-\frac{2}{3 \pi^{2}}
$$

for $N_{c}=N_{f}=3$.

The short-distance behavior of the form factors of both pseudoscalars and axial-vector mesons implies that each individual meson gives a pole contribution with $\bar{\Pi}_{1}\left(Q, Q, Q_{3}\right) \sim Q^{-2} Q_{3}^{-4}$. However, in Refs. [34,35], it was shown that in holographic QCD a summation over the infinite tower of axial-vector mesons changes this. The infinite sum yields

$$
\begin{aligned}
\bar{\Pi}_{1}^{\mathrm{AV}}= & -\frac{g_{5}^{2}}{2 \pi^{4} Q_{3}^{2}} \int_{0}^{z_{0}} d z \int_{0}^{z_{0}} d z^{\prime} \mathcal{J}^{\prime}(Q, z) \mathcal{J}(Q, z) \\
& \times \mathcal{J}^{\prime}\left(Q_{3}, z^{\prime}\right) G_{A}\left(0 ; z, z^{\prime}\right),
\end{aligned}
$$

where $G_{A}$ is the Green's function for the axial-vector mode equation satisfying

$$
\left(z \partial_{z} \frac{1}{z} \partial_{z}-\beta(z)\right) G_{A}\left(0 ; z, z^{\prime}\right)=-z \delta\left(z-z^{\prime}\right)
$$

at $q^{2}=0$. For large $Q, Q_{3} \gg m_{\rho}$, Eq. (46) is dominated by $z, z^{\prime} \ll z_{0}$, where one can approximate $\mathcal{J}(Q, z) \rightarrow$ $Q z K_{1}(Q z)$, and

$$
\begin{aligned}
G_{A}\left(0, z, z^{\prime}\right) & =\frac{1}{2}\left(\min \left(z, z^{\prime}\right)\right)^{2}\left(1+\mathcal{O}\left(Q^{-n}\right)+\mathcal{O}\left(Q_{3}^{-n}\right)\right), \\
n & >0,
\end{aligned}
$$

when $z=\xi / Q$ and $z^{\prime}=\xi^{\prime} / Q_{3}$. This asymptotic behavior of $G_{A}$ holds true in all HW models, including those with a finite quark mass term, because at small $z$, one has $\beta(z) \sim z^{2\left(\Delta_{-}-1\right)}$ with $\Delta_{-}=1$ for the standard choice $M_{X}^{2}=-3$, leading to $n=2$ in Eq. (48); with generalized $M_{X}^{2}$, one has $n>0$ as long as $\Delta_{-}>0$, which corresponds to $M_{X}^{2}<0$. In all cases, we thus obtain for $Q \gg Q_{3} \gg m_{\rho}$ 


$$
\begin{aligned}
\lim _{Q_{3} \rightarrow \infty} \lim _{Q \rightarrow \infty} Q^{2} Q_{3}^{2} \bar{\Pi}_{1}^{\mathrm{AV}}\left(Q, Q, Q_{3}\right) & =-\frac{g_{5}^{2}}{2 \pi^{4}} \int_{0}^{\infty} d \xi \int_{0}^{\infty} d \xi^{\prime} \xi K_{1}(\xi) \frac{d}{d \xi}\left[\xi K_{1}(\xi)\right] \frac{d}{d \xi^{\prime}}\left[\xi^{\prime} K_{1}^{\prime}\left(\xi^{\prime}\right)\right] \frac{1}{2} \xi^{2} \\
& =\frac{g_{5}^{2}}{(2 \pi)^{2}} \frac{1}{2 \pi^{2}} \int_{0}^{\infty} d \xi \xi^{2} \frac{d}{d \xi}\left[\xi K_{1}(\xi)\right]^{2}=-\frac{2}{3 \pi^{2}}
\end{aligned}
$$

for $g_{5}^{2}=12 \pi^{2} / N_{c}=(2 \pi)^{2}$, as required by Eq. (45).

This already implies that in the massive case, the infinite tower of pions (and the other pseudo-Goldstone bosons) should not contribute to the Melnikov-Vainshtein constraint-i.e., summing the infinite number of contributions should not change the asymptotic behavior of the individual contributions in the same way as it happens with axial-vector mesons. In order to check this, we need to analyze

$$
\begin{aligned}
\bar{\Pi}_{1}^{(\pi)}= & -\sum_{n=1}^{\infty} \frac{F_{\pi_{n} \gamma^{*} \gamma^{*}}\left(Q_{1}^{2}, Q_{2}^{2}\right) F_{\pi_{n} \gamma^{*} \gamma}\left(Q_{3}^{2}\right)}{Q_{3}^{2}+m_{n}^{2}}=-\frac{1}{4 \pi^{2}} \sum_{n} \frac{1}{Q_{3}^{2}+m_{n}^{2}}\left\{\mathcal{J}\left(Q_{1}, z_{0}\right) \mathcal{J}\left(Q_{2}, z_{0}\right) \mathcal{J}\left(Q_{3}, z_{0}\right)\left(\frac{z}{\beta} \partial_{z} y_{n}(z)\right)_{z \rightarrow z 0}^{2}\right. \\
& -\mathcal{J}\left(Q_{1}, z_{0}\right) \mathcal{J}\left(Q_{2}, z_{0}\right)\left(\frac{z}{\beta} \partial_{z} y_{n}(z)\right)_{z \rightarrow z 0} \int_{0}^{z_{0}} d z \mathcal{J}\left(Q_{3}, z\right) z y_{n}(z)-\mathcal{J}\left(Q_{3}, z_{0}\right)\left(\frac{z}{\beta} \partial_{z} y_{n}(z)\right)_{z \rightarrow z 0} \\
& \left.\times \int_{0}^{z_{0}} d z \mathcal{J}\left(Q_{1}, z\right) \mathcal{J}\left(Q_{2}, z\right) z y_{n}(z)+\int_{0}^{z_{0}} d z \mathcal{J}\left(Q_{1}, z\right) \mathcal{J}\left(Q_{2}, z\right) z y_{n}(z) \int_{0}^{z_{0}} d z^{\prime} \mathcal{J}\left(Q_{3}, z^{\prime}\right) z^{\prime} y_{n}\left(z^{\prime}\right)\right\} .
\end{aligned}
$$

Since $\lim _{Q \rightarrow \infty} \mathcal{J}\left(Q, z_{0}\right)=0$ and $\lim _{Q \rightarrow \infty} Q^{2} \mathcal{J}\left(Q, z_{0}\right)=0$, the first three terms in the curly brackets do not survive in the large- $Q$ limit. The last term can be formally written as

$$
\begin{array}{r}
-\frac{1}{4 \pi^{2}} \sum_{n=1}^{\infty} \int_{0}^{z_{0}} d z d z^{\prime} \mathcal{J}\left(Q_{1}, z\right) \mathcal{J}\left(Q_{2}, z\right) \mathcal{J}\left(Q_{3}, z^{\prime}\right) z z^{\prime} y_{n}\left(z^{\prime}\right)\left(Q_{3}^{2}-L\right)^{-1} y_{n}(z) \\
=-\frac{1}{4 \pi^{2}} \int_{0}^{z_{0}} d z d z^{\prime} \mathcal{J}\left(Q_{1}, z\right) \mathcal{J}\left(Q_{2}, z\right) \mathcal{J}\left(Q_{3}, z^{\prime}\right) z^{\prime} z\left(Q_{3}^{2}-L\right)^{-1} G\left(z, z^{\prime} ; 0\right),
\end{array}
$$

where $L$ is the differential operator introduced in Eq. (22) and $G$ is its Green's function as defined in Eq. (32). In the limit of interest for the Melnikov-Vainshtein constraint, one has $z^{\prime} \gg z$; thus, Eq. (32) reduces to $\left(L+q^{2}\right)$ $G\left(z, z^{\prime} ; q\right)=0$; hence, effectively $L G\left(z, z^{\prime} ; 0\right)=0$, and we obtain

$$
\begin{aligned}
\left.\bar{\Pi}_{1}^{(\pi)}\right|_{Q \gg Q_{3} \gg m_{\rho}} \rightarrow & -\frac{1}{4 \pi^{2} Q_{3}^{2}} \int_{0}^{z_{0}} d z d z^{\prime} \mathcal{J}\left(Q_{1}, z\right) \mathcal{J}\left(Q_{2}, z\right) \\
& \times \mathcal{J}\left(Q_{3}, z^{\prime}\right) z^{\prime} z G\left(z, z^{\prime} ; 0\right) .
\end{aligned}
$$

At parametrically small $z=\xi / Q$ and $z^{\prime}=\xi^{\prime} / Q_{3}$ and at $q^{2}=0$, Eq. (32) reduces to

$$
\begin{aligned}
\partial_{z} \frac{z}{\beta(z)} \partial_{z} G\left(0 ; z, z^{\prime}\right) & =-\delta\left(z-z^{\prime}\right), \\
\beta(z) & \sim M_{q}^{2}\left(z / z_{0}\right)^{2-2 \Delta_{-},}
\end{aligned}
$$

provided $\Delta_{-}>0$.

In the massive HW models with $M_{X}^{2}=-3$, this gives

$$
G\left(0 ; z, z^{\prime}\right) \rightarrow-M_{q}^{2} \ln \left(\max \left(z, z^{\prime}\right)\right)+\text { const }
$$

leading to

$$
\begin{aligned}
& -Q^{2} Q_{3}^{2} \bar{\Pi}_{1}^{(\pi)}\left(Q, Q, Q_{3}\right) \\
& \quad \sim \frac{M_{q}^{2}}{4 \pi^{2}} \frac{\ln \left(Q_{3}\right)}{Q_{3}^{2}} \int_{0}^{\infty} d \xi \xi^{3}\left[K_{1}(\xi)\right]^{2} \int_{0}^{\infty} d \xi^{\prime} \xi^{\prime 2} K_{1}\left(\xi^{\prime}\right) \\
& =\frac{M_{q}^{2}}{6 \pi^{2}} \frac{\ln \left(Q_{3}^{2}\right)}{Q_{3}^{2}} \rightarrow 0
\end{aligned}
$$

for large $Q \gg Q_{3} \gg m_{\rho}$. Thus, the summation of the infinite tower of pions does give a different asymptotic behavior from that of individual contributions, but only in the form of a logarithmic enhancement.

In Fig. 3, the contributions of the first four pion modes to $P_{1} \equiv-Q^{2} Q_{3}^{4} \bar{\Pi}_{1}^{(\pi)}\left(Q, Q, Q_{3}\right)$-i.e., the left-hand side of Eq. (55) with another factor of $Q_{3}^{2}$-are plotted for the HW1m model with $Q=200 \mathrm{GeV}$ and increasing $Q_{3}$. Numerically, this is dominated by the contribution from the lightest pion, which completely swamps the logarithmic term in Eq. (55), whose prefactor $M_{q}^{2} /\left(3 \pi^{2}\right)$ is of the order $\sim 3 \times 10^{-6} \mathrm{GeV}^{2}$. Note that this term is suppressed by an extra power of quark mass compared to the contribution from the magnetic susceptibility [55,71] to the asymptotic behavior of $\bar{\Pi}_{1}$ worked out in Ref. [23]. In Fig. 4, the same is plotted with quark masses increased by a factor of 25 

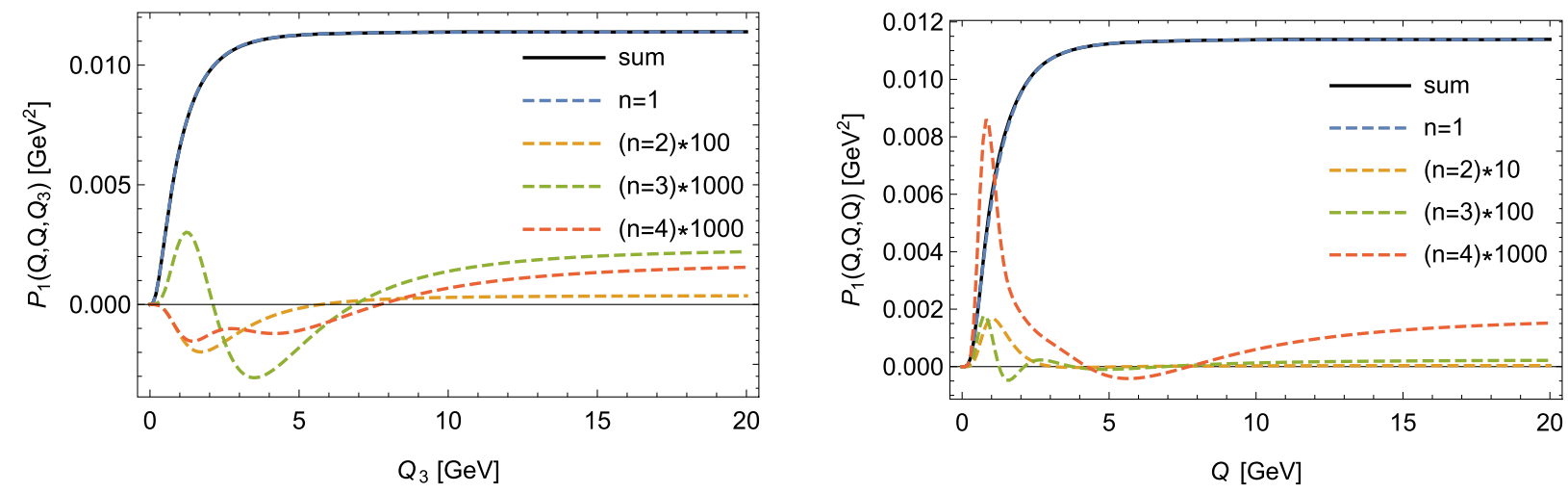

FIG. 3. Plot of the contributions of the first four pion modes to $P_{1}\left(Q, Q, Q_{3}\right)=-Q^{2} Q_{3}^{4} \Pi_{1}^{(\pi)}\left(Q, Q, Q_{3}\right)$ for the HW1m model with $Q=200 \mathrm{GeV}$ in the left panel and $Q_{3}=Q$ in the right panel, showing that the contribution from the tower of pseudoscalars to the LSDC is suppressed like $1 / Q_{3}^{2}$ up to (here invisible) logarithmic corrections.
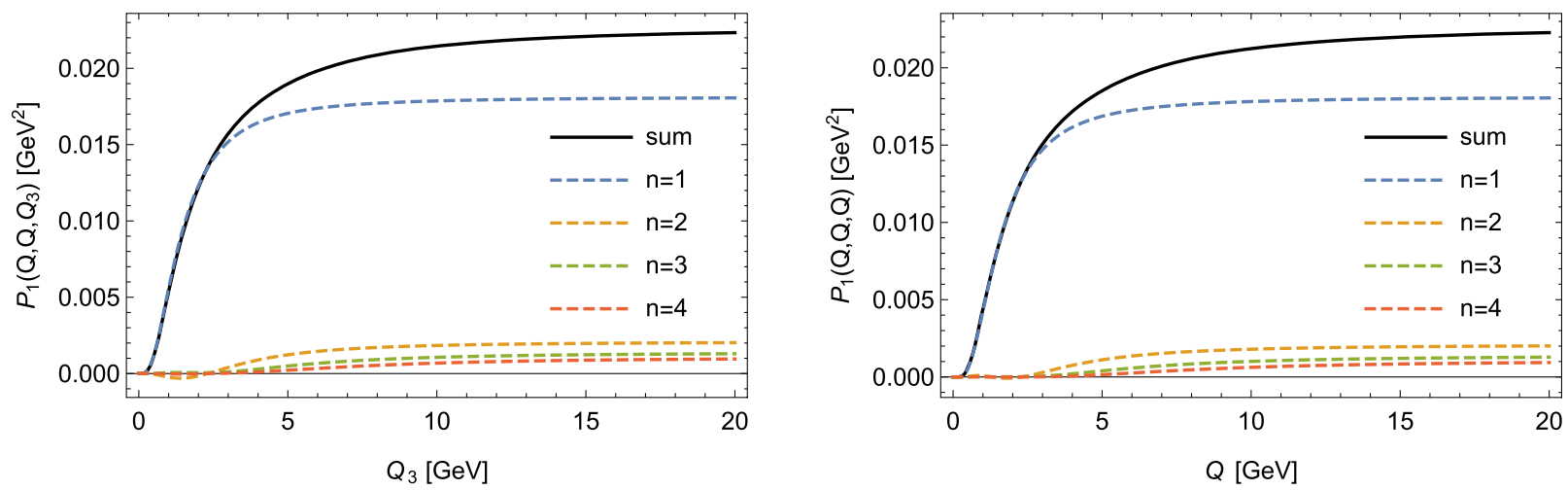

FIG. 4. As in Fig. 3, but with quark masses increased by a factor of 25, illustrating the buildup of a logarithmic enhancement by the summation of the infinite tower of massive pions.

(corresponding to a pion mass of about $750 \mathrm{MeV}$ ), where the slow buildup of a logarithmic term becomes apparent.

When $M_{X}^{2}<-3$, the logarithmic enhancement disappears, and $Q^{2} Q_{3}^{2} \bar{\Pi}_{1}^{(\pi)}\left(Q, Q, Q_{3}\right) \sim Q_{3}^{-2}$. For $-3<M_{X}^{2}<0$, where $1>\Delta_{-}>0$, one instead obtains a power-law enhancement from

$$
G\left(0 ; z, z^{\prime}\right) \rightarrow \frac{M_{q}^{2}}{2\left(1-\Delta_{-}\right)}\left(\frac{z_{0}}{z^{\prime}}\right)^{2\left(1-\Delta_{-}\right)} \propto Q_{3}^{2\left(1-\Delta_{-}\right)}
$$

such that

$$
Q^{2} Q_{3}^{2} \bar{\Pi}_{1}^{(\pi)}\left(Q, Q, Q_{3}\right) \sim-\frac{2^{2 \Delta^{-}-3} \Gamma\left(\Delta^{-}\right) \Gamma\left(1+\Delta^{-}\right)}{3 \pi^{2}} \frac{M_{q}^{2}}{Q_{3}^{2}} \frac{\left.\left(z_{0} Q_{3}\right)^{2\left(1-\Delta^{-}\right.}\right)}{1-\Delta^{-}} \propto Q_{3}^{-2 \Delta^{-}} .
$$

Only for $\Delta_{-}=0$, which is at the border of the allowed range $M_{X}^{2} \in(-4,0)$, would the infinite tower of massive pions start to contribute to the Melnikov-Vainshtein constraint, exactly when the result of Eq. (49) for the infinite tower of axial-vector mesons would break down. However, in the following applications, the phenomenologically interesting generalizations of $M_{X}^{2}$ all have $M_{X}^{2}<-3$, where the contribution from the pseudoscalar tower to the longitudinal short-distance constraint is suppressed by two inverse powers of $Q_{3}$ without even a logarithmic enhancement.

In the chiral limit, the massive pions still contribute to $\bar{\Pi}_{1}$, even though they decouple from the axial-vector current and from the axial anomaly. With strictly $M_{q}=0$, 


$$
\begin{aligned}
G\left(0 ; z, z^{\prime}\right)_{\text {chiral }} & \rightarrow \text { const }+\frac{\Sigma^{2} z_{0}^{4}}{2\left(1-\Delta_{+}\right)}\left(\frac{z_{0}}{z^{\prime}}\right)^{2\left(1-\Delta_{+}\right)} \\
& \propto \text { const }+Q_{3}^{-2-2 \alpha}
\end{aligned}
$$

so in this case there is no enhancement from the summation of the infinite tower, irrespective of the value of $M_{X}^{2}$.

$$
\text { 2. } Q_{1}^{2}=Q_{2}^{2}=Q_{3}^{2} \gg m_{\rho}^{2}
$$

In the symmetric limit $Q_{1}^{2}=Q_{2}^{2}=Q_{3}^{2} \gg m_{\rho}^{2}$, operator product expansion and LO $\mathrm{pQCD}$ imply that the longitudinal short-distance constraint is $2 / 3$ of the value appearing in Eq. (45) [8,23]:

$$
\lim _{Q \rightarrow \infty} \lim _{Q \rightarrow \infty} Q^{4} \bar{\Pi}_{1}(Q, Q, Q)=-\frac{4}{9 \pi^{2}}
$$

In this case, the same derivation as above leads to

$$
\begin{aligned}
\lim _{Q \rightarrow \infty} Q^{4} \bar{\Pi}_{1}^{\mathrm{AV}}(Q, Q, Q) & =-\frac{g_{5}^{2}}{2 \pi^{4}} \int_{0}^{\infty} d \xi \int_{0}^{\infty} d \xi^{\prime} \frac{1}{2} \frac{d}{d \xi}\left[\xi K_{1}(\xi)\right]^{2} \frac{d}{d \xi^{\prime}}\left[\xi^{\prime} K_{1}^{\prime}\left(\xi^{\prime}\right)\right] \frac{1}{2}\left[\min \left(\xi, \xi^{\prime}\right)\right]^{2} \\
& =-\frac{g_{5}^{2}}{(2 \pi)^{2} \pi^{2}} \int_{0}^{\infty} d \xi \int_{0}^{\infty} d \xi^{\prime}\left[\xi K_{1}(\xi)\right]^{2}\left[\xi^{\prime} K_{1}^{\prime}\left(\xi^{\prime}\right)\right] \xi \delta\left(\xi-\xi^{\prime}\right) \\
& =-\frac{g_{5}^{2}}{(2 \pi)^{2} \pi^{2}} \int_{0}^{\infty} d \xi \xi\left[\xi K_{1}(\xi)\right]^{3}=-0.361 \frac{g_{5}^{2}}{(2 \pi)^{2} \pi^{2}},
\end{aligned}
$$

which reproduces the correct power behavior, but for $g_{5}=2 \pi$, as demanded by Eq. (14), the numerical value is only $81 \%$ of the result in Eq. (59). Again, this result for the contribution of the infinite tower of axial-vector mesons is the same for chiral and for massive HW models.

The asymptotic contribution of the pseudoscalar tower is still given by the expression (51), but the argument given thereafter does not apply in the symmetric limit. However, numerically we found no evidence of an enhancement of the asymptotic behavior due to the summation of the infinite tower of massive pions beyond what is seen in the asymmetric case-see Figs. 3 and 4.

\section{NUMERICAL RESULTS}

In the following, we compare the different HW models numerically, in particular with regard to the contribution of the infinite tower of massive pions to the hadronic light-bylight scattering amplitude, and thereby to the anomalous magnetic moment of the muon.

In all models, we have chosen $g_{5}=2 \pi$, ensuring an exact fit of the asymptotic LO pQCD result [Eq. (14)], but in the later discussion we shall also consider relaxing this constraint to account for the fact that at any large but finite energy scale, there is a non-negligible reduction of TFFs of the order $\alpha_{s} / \pi$ that in AdS/QCD could perhaps be simply ${ }^{3}$ modeled by a small reduction of $g_{5}$. The low-energy parameters $f_{\pi}$ and $m_{\rho}$ are always fitted to their physical

\footnotetext{
${ }^{3}$ More elaborate holographic QCD models would do this by a modification of the anti-de Sitter background.
}

values, fixed to $92.4 \mathrm{MeV}$ and $775 \mathrm{MeV}$, respectively, to be consistent with our previous work [34].

We consider two possibilities (HW1, HW3) for boundary conditions at $z=z_{0}$ [always given by Eq. (11)], and also alternatively the standard choice $M_{X}^{2}=-3$ and having $M_{X}^{2}$ as a tunable parameter. We thus employ four different HW models with nonvanishing light-quark masses:

HW1m is the direct extension of the chiral HW1 model employed in our previous studies [34,41]. It coincides with model A of Erlich, Katz, Son, and Stephanov [38], except that we have fitted to the mass of the neutral instead of the charged pions.

HW1 $\mathbf{m}^{\prime}$ deviates from the standard choice $M_{X}^{2}=-3$ in order to attempt a better fit of the masses of the first excited pion and/or the lightest axial-vector meson. It turns out that only the latter can be matched to the $a_{1}$ mass. The mass of the first excited pion is then also reduced compared to the pristine HW1 model, from around 1900 to $1600 \mathrm{MeV}$, but the target of $1300 \mathrm{MeV}$ cannot be reached.

HW3m uses the standard choice $M_{X}^{2}=-3$, but with boundary conditions as proposed in Ref. [51], which have the advantage of making a manual subtraction of infrared boundary contributions in the Chern-Simons action unnecessary.

HW3 $\mathbf{m}^{\prime}$ uses additionally $M_{X}^{2}$ as a free parameter, which in this model achieves a fit of the mass of the first excited pion of $1300 \mathrm{MeV}$, as was the main motivation put forward by Domènech, Panico, and Wulzer in Ref. [51] for proposing this kind of model. Our HW $3 \mathrm{~m}^{\prime}$ slightly deviates from the parameters used in Ref. [51], because we fitted $f_{\pi}, m_{\rho}, m_{\pi^{0}}$, and 
TABLE I. Numerical results for various HW models (in the chiral HW1 model with the pion mass raised manually ( $户$ ) to $135 \mathrm{MeV}$ in the evaluation of $a_{\mu}$ ). Fitted values are marked by $*$. The axial-vector contributions correspond to a whole $U\left(N_{f}=3\right)$ multiplet, which in the present flavor-symmetric case is simply $a_{\mu}^{A_{n}}=a_{\mu}^{\left(a_{1}+f_{1}+f_{1}^{\prime}\right)_{n}}=4 a_{\mu}^{a_{1 n}}$.

\begin{tabular}{|c|c|c|c|c|c|c|c|c|c|c|}
\hline Model & PS & $n=1$ & $n=2$ & $n=3$ & AV & $n=1$ & $n=2$ & $n=3$ & $n=4$ & $n=5$ \\
\hline HW1 chiral & $m_{\pi_{n}}[\mathrm{MeV}]$ & $0 \gg 135$ & 1899 & 2887 & $m_{\mathrm{A}_{n}}[\mathrm{MeV}]$ & 1375 & 2154 & 2995 & 3939 & 4917 \\
\hline \multirow[t]{3}{*}{$M_{X}^{2}=-3$} & $f_{\pi_{n}}[\mathrm{MeV}]$ & $92.4 *$ & 0 & 0 & $F_{\mathrm{A}_{n}} / m_{\mathrm{A}_{n}}[\mathrm{MeV}]$ & 177 & 204 & 263 & 311 & 351 \\
\hline & $F_{\pi_{n} \gamma \gamma}\left[\mathrm{GeV}^{-1}\right]$ & 0.274 & -0.202 & 0.153 & $A(0,0)\left[\mathrm{GeV}^{-2}\right]$ & -21.04 & -2.93 & 0.294 & -2.16 & 0.400 \\
\hline & $a_{\mu}^{\pi_{n}} \times 10^{11}$ & 65.2 & 0.7 & 0.1 & $a_{\mu}^{\mathrm{A}_{n}} \times 10^{11}$ & 31.4 & 4.7 & 1.8 & 1.2 & 0.5 \\
\hline HW1m & $m_{\pi_{n}}[\mathrm{MeV}]$ & $135^{*}$ & 1892 & 2882 & $m_{\mathrm{A}_{n}}[\mathrm{MeV}]$ & 1367 & 2141 & 2987 & 3934 & 4914 \\
\hline \multirow{3}{*}{$M_{X}^{2}=-3$} & $f_{\pi_{n}}^{n}[\mathrm{MeV}]$ & $92.4^{*}$ & 1.56 & 1.25 & $F_{\mathrm{A}_{n}} / m_{\mathrm{A}_{n}}[\mathrm{MeV}]$ & 175 & 204 & 263 & 311 & 351 \\
\hline & $F_{\pi_{n} \gamma \gamma}\left[\mathrm{GeV}^{-1}\right]$ & 0.276 & -0.203 & 0.154 & $A(0,0)\left[\mathrm{GeV}^{-2}\right]$ & -21.00 & -3.21 & 0.328 & -2.16 & 0.376 \\
\hline & $a_{\mu}^{\pi_{n}} \times 10^{11}$ & 66.0 & 0.7 & 0.1 & $a_{\mu}^{\mathrm{A}_{n}} \times 10^{11}$ & 31.4 & 4.9 & 1.8 & 1.2 & 0.5 \\
\hline HW1m' & $m_{\pi_{n}}[\mathrm{MeV}]$ & $135^{*}$ & 1591 & 2564 & $m_{\mathrm{A}_{n}}[\mathrm{MeV}]$ & $1230^{*}$ & 1977 & 2901 & 3879 & 4873 \\
\hline \multirow[t]{3}{*}{$M_{X}^{2}=-3.837$} & $f_{\pi_{n}}[\mathrm{MeV}]$ & $92.4 *$ & 1.59 & 0.950 & $F_{\mathrm{A}_{n}} / m_{\mathrm{A}_{n}}[\mathrm{MeV}]$ & 148 & 208 & 266 & 312 & 351 \\
\hline & $F_{\pi_{n} \gamma \gamma}\left[\mathrm{GeV}^{-1}\right]$ & 0.277 & -0.250 & 0.194 & $A(0,0)\left[\mathrm{GeV}^{-2}\right]$ & -19.95 & -7.29 & 0.678 & -2.18 & 0.341 \\
\hline & $a_{\mu}^{\pi_{n}} \times 10^{11}$ & 64.3 & 1.5 & 0.3 & $a_{\mu}^{\mathrm{A}_{n}} \times 10^{11}$ & 29.8 & 8.7 & 2.0 & 1.3 & 0.5 \\
\hline HW3m & $m_{\pi_{n}}[\mathrm{MeV}]$ & $135^{*}$ & 1715 & 2513 & $m_{\mathrm{A}_{n}}[\mathrm{MeV}]$ & 1431 & 2421 & 3398 & 4387 & 5384 \\
\hline \multirow[t]{3}{*}{$M_{X}^{2}=-3$} & $f_{\pi_{n}}[\mathrm{MeV}]$ & $92.4 *$ & 1.56 & 1.34 & $F_{\mathrm{A}_{n}} / m_{\mathrm{A}_{n}}[\mathrm{MeV}]$ & 195 & 244 & 291 & 332 & 369 \\
\hline & $F_{\pi_{n} \gamma \gamma}\left[\mathrm{GeV}^{-1}\right]$ & 0.277 & -0.196 & 0.0797 & $A(0,0)\left[\mathrm{GeV}^{-2}\right]$ & -21.27 & 0.310 & -2.09 & -0.299 & -0.514 \\
\hline & $a_{\mu}^{\pi_{n}} \times 10^{11}$ & 66.6 & 0.8 & 0.04 & $a_{\mu}^{\mathrm{A}_{n}} \times 10^{11}$ & 32.7 & 3.4 & 1.7 & 0.7 & 0.4 \\
\hline $\mathrm{HW} 3 \mathrm{~m}^{\prime}$ & $m_{\pi_{n}}[\mathrm{MeV}]$ & $135^{*}$ & $1300^{*}$ & 2113 & $m_{\mathrm{A}_{n}}[\mathrm{MeV}]$ & 1380 & 2355 & 3345 & 4345 & 5350 \\
\hline \multirow[t]{3}{*}{$M_{X}^{2}=-3.841$} & $f_{\pi_{n}}[\mathrm{MeV}]$ & $92.4 *$ & 1.92 & 1.29 & $F_{\mathrm{A}_{n}} / m_{\mathrm{A}_{n}}[\mathrm{MeV}]$ & 186 & 242 & 291 & 332 & 369 \\
\hline & $F_{\pi_{n} \gamma \gamma}\left[\mathrm{GeV}^{-1}\right]$ & 0.278 & -0.206 & 0.0474 & $A(0,0)\left[\mathrm{GeV}^{-2}\right]$ & -21.29 & -0.841 & -1.76 & -0.440 & -0.476 \\
\hline & $a_{\mu}^{\pi_{n}} \times 10^{11}$ & 66.0 & 1.5 & 0.01 & $a_{\mu}^{\mathrm{A}_{n}} \times 10^{11}$ & 33.2 & 4.1 & 1.8 & 0.8 & 0.4 \\
\hline
\end{tabular}

$m_{\pi(1300)}$ instead of performing a least-squares fit over a larger set of low-energy parameters.

For the chiral limit, we only consider the HW1 model, where we update the results obtained for pions in Ref. [41] $]^{4}$ and recapitulate the results for axial-vector mesons in Ref. [34].

\section{A. Masses}

As Table I shows, in the chiral HW1 model, the mass of the lightest axial-vector multiplet is above the physical masses [72] $M_{a_{1}(1260)}=1230(40) \mathrm{MeV}$ and $M_{f_{1}(1285)}=$ $1281.9(5) \mathrm{MeV}$, but below that of the $f_{1}^{\prime}, M_{f_{1}(1420)}=$ 1426.3(9) MeV. While this prediction of the HW1 model is in the right ballpark, the mass of the first excited pion is $1899 \mathrm{MeV}$, and thus significantly above $M_{\pi(1300)}=1300 \pm 100 \mathrm{MeV}$.

With nonzero light-quark masses, the HW1m model has slightly reduced axial-vector meson mass and increased excited pion masses. In the HW1 $\mathrm{m}^{\prime}$ model, where the $a_{1}$ mass can be matched, the lowest excited pion mass is reduced to about $1600 \mathrm{MeV}$.

\footnotetext{
${ }^{4}$ Note that the original version of Ref. [41] contained an error in the $a_{\mu}$ results for the HW1 model, but not in the plots of the corresponding TFFs.
}

With HW3 boundary conditions, there is additional chiral symmetry breaking, and therefore a larger difference between the vector and axial-vector meson masses, so that $m_{a_{1}}$ is now even above the mass of the physical $f_{1}^{\prime}$, but the excited pion mass is lowered substantially compared to the HW1m model, while still being too high. In the HW3 $\mathrm{m}^{\prime}$ model, where the first excited pion can be brought down to $1300 \mathrm{MeV}$, the axial-vector meson mass is then also lowered, but it remains somewhat larger than in the HW1 models. Even in the HW3 $\mathrm{m}^{\prime}$ model, where the pseudoscalar masses are the smallest, the second excited $(n=3)$ pion has a mass higher than the next established pion state $\pi(1800)$ and instead close to the next (less established) state $\pi(2070)$; in the other models, $m_{n=3}$ is far higher.

\section{B. Decay constants}

The results for the decay constant of the lowest excited pion in the massive HW models span the range $(1.56 \ldots 1.92) \mathrm{MeV}$, with the $\mathrm{HW} 3 \mathrm{~m}^{\prime}$ model, where the mass of $\pi(1300)$ can be fitted, yielding the largest value. These values are well below the existing experimental upper bound of $8.4 \mathrm{MeV}$ [73]; the result for the HW3m'

\footnotetext{
${ }^{5}$ Note, however, that this state is sometimes considered to be a non- $q \bar{q}$ state [72].
} 
model is actually fully consistent with the value 2.20(46) MeV of Ref. [74] that was adopted in Ref. [25]. ${ }^{6}$

The holographic results for the decay constant of the lightest axial-vector meson, defined in analogy to Eq. (13), read $F_{a_{1}}=(493 \mathrm{MeV})^{2}$ in the chiral HW1 model and $(426 \ldots . .506 \mathrm{MeV})^{2}$ for the different massive HW models. Note that in the literature, frequently the mass of the axial-vector meson is factored out [77]. ${ }^{7}$ In Ref. [78], a value of $F_{a_{1}} / m_{a_{1}}=168(7) \mathrm{MeV}$ has been obtained from light-cone sum rules. With $F_{a_{1}} / m_{a_{1}}=$ $177 \mathrm{MeV}$ for the chiral HW1 model and (148... 195) $\mathrm{MeV}$ for the different massive HW models (see Table I), the ballpark spanned by the holographic results is broadly consistent with that.

\section{Comparison of transition form factors}

In Figs. 5 and 6, the single- and double-virtual pion TFFs following from the chiral and the massive HW models are compared with each other and with the results obtained in Ref. [22] in the dispersive approach [70]. The HW results all lie within the error band of the dispersive result, mostly above its central value, with only $\mathrm{HW} 1 \mathrm{~m}^{\prime}$ below.

Table I also lists the amplitude $F_{\pi_{n} \gamma \gamma}$ for pseudoscalar decays into two real photons. For the ground-state pion, there is only a tiny change when finite quark masses are introduced, across all massive HW models. For excited pions, there is more variation; for the first excited pion, the range of $\left|F_{\pi_{2} \gamma \gamma}\right|$ is $(0.196 \ldots 0.250) \mathrm{GeV}^{-1}$. In the HW3m' model, where one can fit to $m_{2}=1300 \mathrm{MeV}$, the value is $0.206 \mathrm{GeV}^{-1}$. At present, no direct measurements are available, but data on certain branching ratios permit one to derive an estimate for an upper bound [25] reading $\left|F_{\pi(1300) \gamma \gamma}\right|<0.0544(71) \mathrm{GeV}^{-1}$. Evidently, the holographic results strongly overestimate the two-photon coupling of the first excited pion. This could be taken as a hint that it is better to choose a model where the masses of the lightest axial-vector mesons can be fitted to experiment—namely, the HW1 $\mathrm{m}^{\prime}$ model-since the twophoton couplings of axial-vector mesons play a more important role altogether. The holographic results obtained for the whole tower of excited pions could still be taken as a rough estimate of (perhaps an upper bound of) this contribution in real QCD. It is certainly also conceivable that the contributions of individual modes are overestimated while their combined contribution is closer

\footnotetext{
${ }^{6}$ The decay constants of the higher pion modes fall off with increasing mode number $n$, inversely proportional to $m_{n}^{x}$, where $x$ is smaller than 1 , which is very different from the value $n=2$ of Ref. [75] and closer to but still not agreeing with Ref. [76], where $x=1$. However, the HW models lack linear Regge trajectories; soft-wall models may be more realistic here.

${ }^{7}$ Our definition of $F_{a_{1}}$ corresponds to $F_{A}^{a=3} m_{A}$ and $F_{A}^{a=3} m_{A} / \sqrt{2}$ in Refs. [63,69] and Ref. [78], respectively.
}

to real QCD, where the mass spectrum of excited pions is denser than in the HW models.

Table I also shows that the chiral HW1 model has almost identical results for $F_{\pi_{n} \gamma \gamma}$ to the HW1m model. As emphasized in Ref. [25], the vanishing of the decay constants of massive pions in the chiral limit does not preclude a coupling to photons. The former only means decoupling of the massive pions from the axial-vector current and the axial anomaly. Thus, massive pions contribute to the HLBL scattering amplitude also in the chiral HW1 model, and therefore to the anomalous magnetic moment of the muon. ${ }^{8}$

Figure 7 displays the single- and double-virtual TFFs for the first excited pion in the various HW models. Also shown are the corresponding quantities in the Regge model of Refs. [24,25] (RM) and its modification according to Appendix E therein $\left(\mathrm{RM}^{\prime}\right)$. The Regge model TFFs satisfy the experimental upper bound $F_{\pi(1300) \gamma \gamma}$, but they do not obey the single-virtual Brodsky-Lepage limit with the decay constants of excited pseudoscalars. The HW results have a much larger amplitude at vanishing virtualities, but decay faster. They have a zero and a sign change before approaching the asymptotic behavior [Eq. (41)]. The TFFs of higher excited pions have more zeros and reduced asymptotic limits due to smaller decay constants.

In Fig. 8, the shape of the axial-vector TFF $A\left(Q_{1}^{2}, Q_{2}^{2}\right) / A(0,0)$ is shown in the single-virtual and in the symmetric double-virtual cases. In the single-virtual case, we also show the experimental result obtained in Ref. [42] for the $f_{1}(1285)$ meson, which is found to be remarkably compatible, in particular for the $\mathrm{HW} 1 \mathrm{~m}^{\prime}$ model, where the mass of the lightest axial-vector meson can be fitted.

The normalization factor $A(0,0)$ is directly related to the so-called equivalent two-photon rate [67,79]. In Ref. [34], we have compared with a combination of the experimental results obtained for the $f_{1}(1285)$ and $f_{1}(1420)$ mesons. Since we are actually using flavor-symmetric models, one should account for $\mathrm{SU}(3)$ flavor breaking ${ }^{9}$ before matching $A(0,0)$, which leads to $|A(0,0)|_{f_{1}(1285)}^{\exp }=16.6(1.5) \mathrm{GeV}^{-2}$. In Refs. [37,68], the corresponding value for the lightest $a_{1}$ meson has been estimated as $|A(0,0)|_{a_{1}(1260)}^{\exp }=$ 19.3(5.0) $\mathrm{GeV}^{-2}$. In the HW models, we find the range $|A(0,0)|_{n=1}=(19.95 \ldots 21.29) \mathrm{GeV}^{-2}$, which is compatible with the latter, but above the value obtained for the $f_{1}(1285)$ meson.

\footnotetext{
${ }^{8}$ The simpler HW2 model considered in Refs. [34,35] does not involve a bifundamental bulk scalar field, and thus does not have excited pion states at all.

${ }^{9}$ We are grateful to Martin Hoferichter for pointing this out to us.
} 

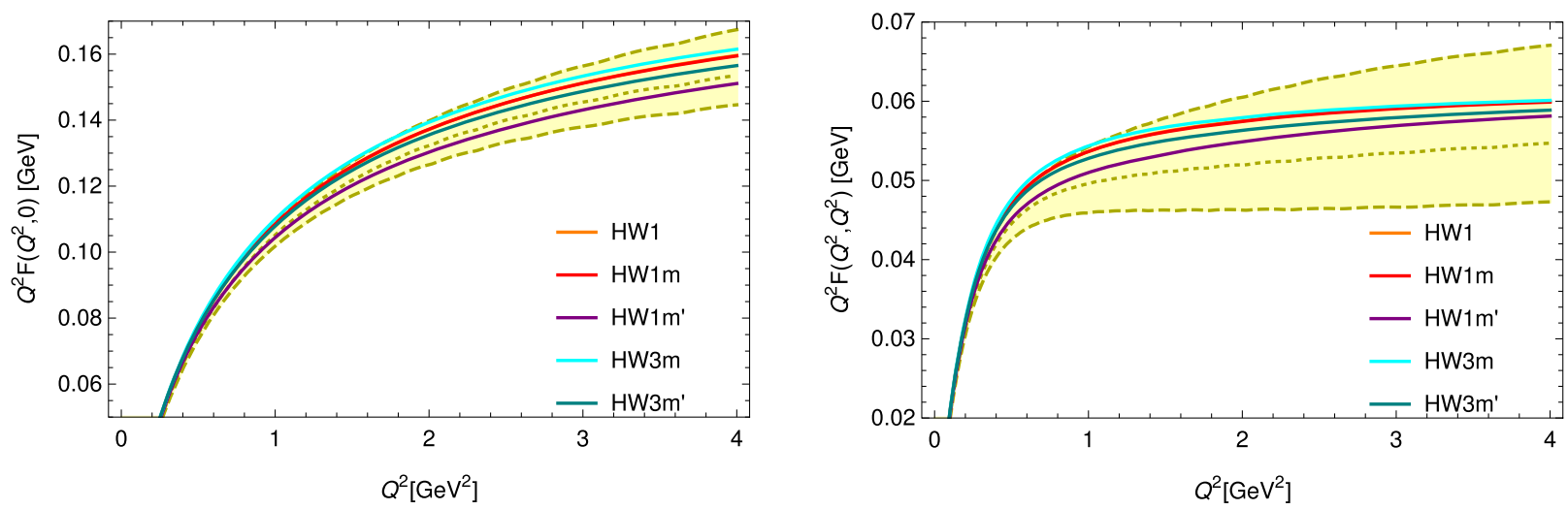

FIG. 5. Single- and double-virtual pion transition form factors in the various HW models together with the result of the data-driven dispersive approach of Ref. [22] (yellow band for the estimated error with dotted dark-yellow points for the central result).
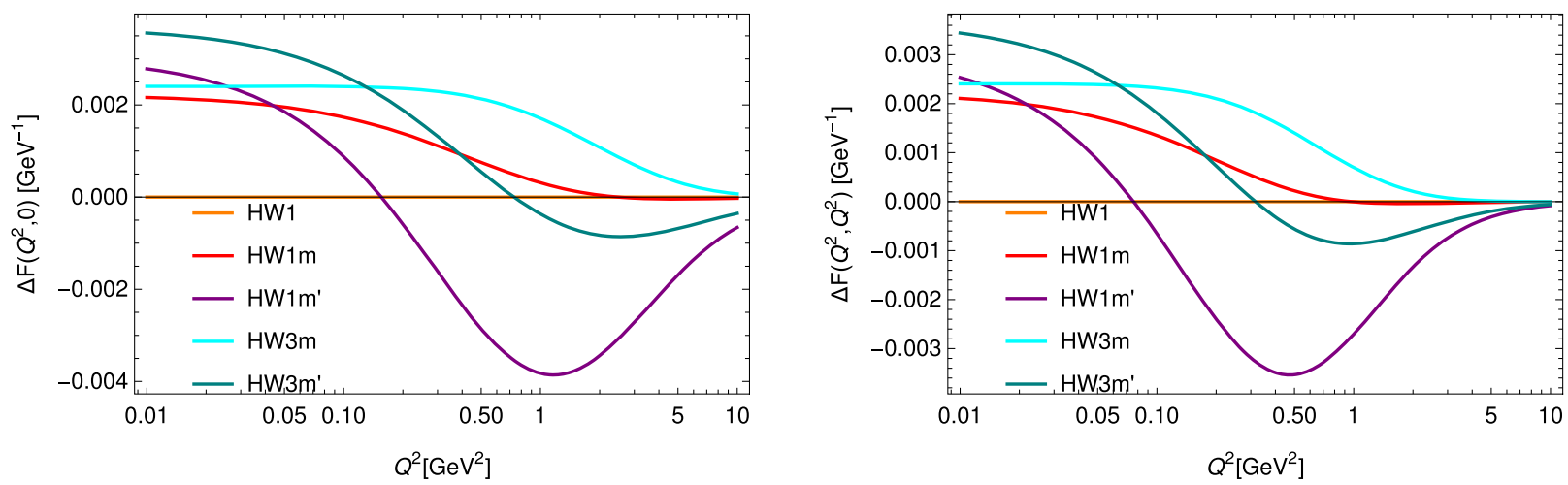

FIG. 6. Deviations of the single- and double-virtual pion transition form factors in the massive HW models from the chiral HW1 model.
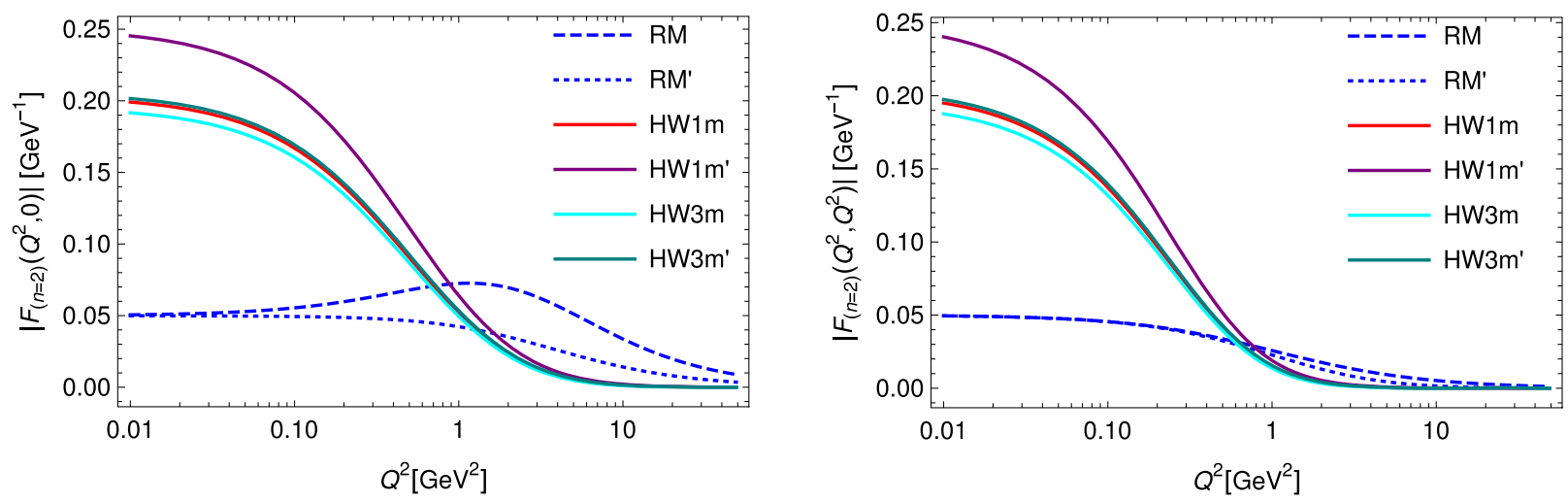

FIG. 7. Single- and double-virtual TFFs for the first excited pion in the various HW models. Also given are the corresponding quantities in the Regge model of Refs. [24,25] (RM) and its modification according to Appendix E therein $\left(\mathrm{RM}^{\prime}\right)$.

\section{HLBL contribution to $a_{\mu}$}

In Table I, we also give the holographic results for the contributions to $a_{\mu}$, the anomalous magnetic moment of the muon, from the first few states of the pion and axial-vector meson towers; in Fig. 9, the results for the $\pi^{0}$ and $a_{1}$ sector are shown in the form of a bar chart.
In the chiral HW1 model, the chiral TFFs for the pion have been combined with a pion propagator where the physical mass of $\pi^{0}$ has been inserted by hand. The result of $a_{\mu}^{\pi^{0}}=65.2 \times 10^{-11}$ is remarkably close to that obtained in the massive HW models, which together span the range $a_{\mu}^{\pi^{0}}=(64.3 \ldots 66.6) \times 10^{-11}$. The results of the massive 

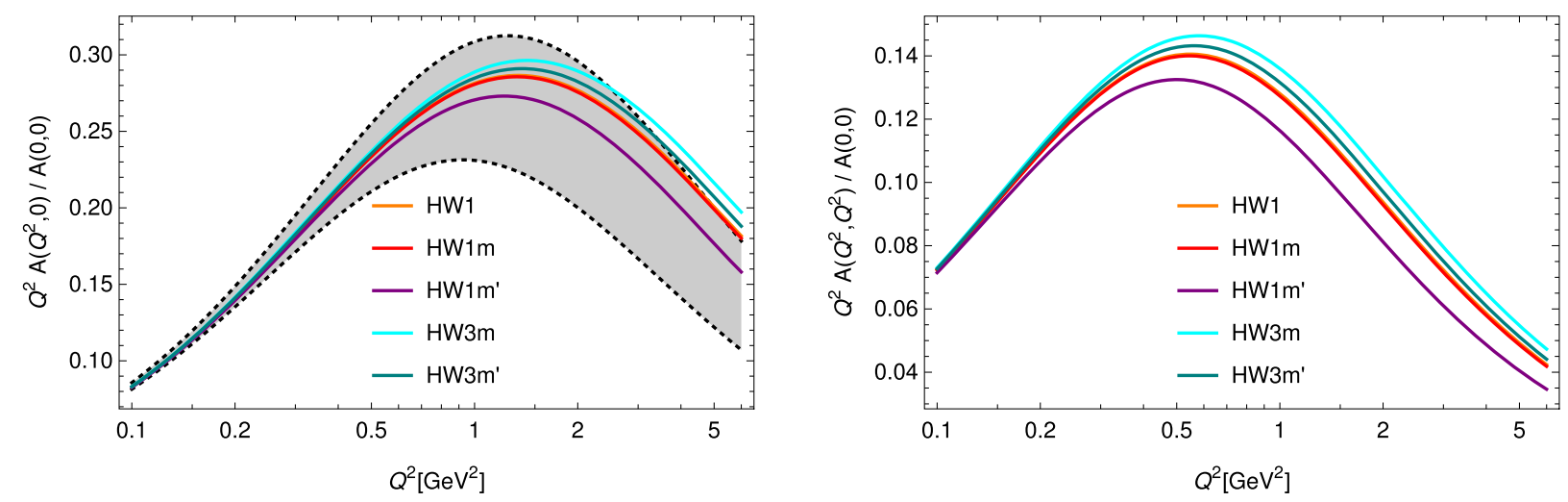

FIG. 8. Single- and double-virtual axial-vector transition form factors, the former compared with the dipole fit of L3 data for $f_{1}(1285)$ of Ref. [42] (gray band).

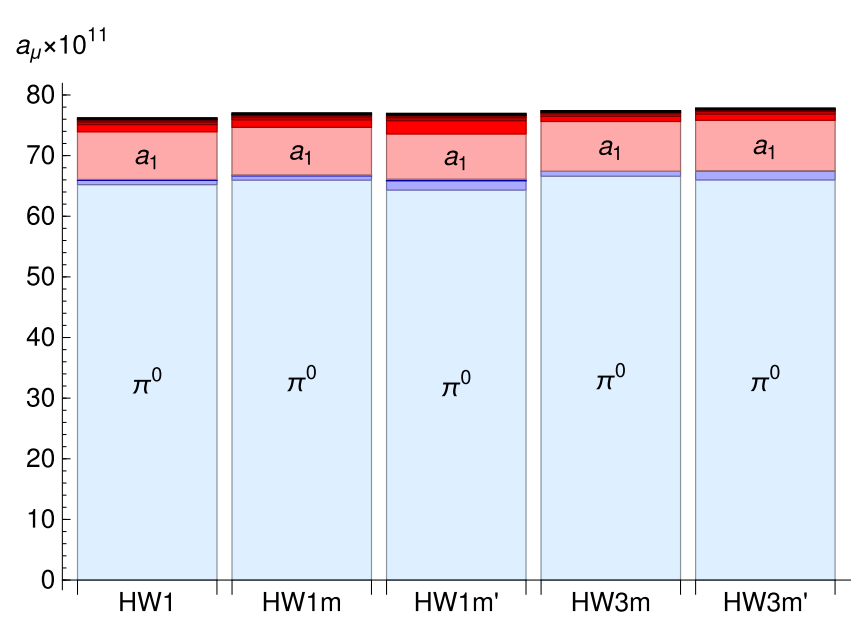

FIG. 9. Bar chart of the individual contributions to $a_{\mu}^{\pi \cup a_{1}}$ in the various HW models, with excited modes given by increasingly darker colors, with blue for the $\pi^{0}$ 's and red for the $a_{1}$ 's.

HW1m model are very close to those of the chiral HW1 model, and also for the other contributions. Somewhat more variation is obtained in the other models, where either different boundary conditions or different values of $M_{X}^{2}$ are employed.

Table II shows the sums of the contributions in the different sectors, where we have made a numerical estimate of the limit value when the infinite tower of axial-vector mesons is included as in Ref. [34]; in the case of the excited pions, the contributions of the higher modes fall off very quickly, so we have just summed the first few modes.

For the contributions of the excited pions, we obtain the range $a_{\mu}^{\pi^{*}}=(0.8 \ldots 1.8) \times 10^{-11}$, with the chiral model being at the lower end. Even though we have seen above that the HW models appear to severely overestimate the two-photon coupling $F_{\pi_{2} \gamma \gamma}$ when comparing with the upper limit [25] $\left|F_{\pi(1300) \gamma \gamma}\right|$ given above, the holographic results are somewhat below the contributions from the first few excited states obtained in Ref. [25] with large- $N_{c}$ Regge models. ${ }^{10}$ In Ref. [41] also, the contributions from the $\eta$ and $\eta^{\prime}$ pseudoscalars were estimated on the basis of the chiral HW1 model; we defer a precise evaluation of those in the massive HW models to future work, where we plan to study the flavor-asymmetric case together with the contributions from the Witten-Veneziano mechanism for implementing the $U(1)_{A}$ anomaly. In the present flavor-symmetric setup, we would simply estimate the contribution of a whole $U\left(N_{f}=3\right)$ multiplet $\left(P^{*}\right)$ as $a_{\mu}^{\mathrm{P}^{*}} \equiv 4 a_{\mu}^{\pi^{*}}=(3.4 \ldots 7.2) \times 10^{-11}$.

The much higher contributions from the infinite tower of axial-vector mesons, which in the chiral HW1 model reads [34] $a_{\mu}^{\mathrm{A}}=40.6 \times 10^{-11}$, span the range $(39.3 \ldots 43.3) \times$ $10^{-11}$ in the massive HW models, where the highest value is obtained in the HW1 $\mathrm{m}^{\prime}$ model, in which the physical mass of the $a_{1}$ meson can be fitted by using a nonstandard $M_{X}$ value. This model has also the largest contribution from the excited pseudoscalars, so that in combination $a_{\mu}^{\mathrm{A}+\mathrm{P}^{*}}=50.5 \times 10^{-11}$ is reached; the lower end of the results for this quantity is provided by the HW3m model with $43.3 \times 10^{-11}$, where the masses of axial-vector mesons are in fact too high overall.

Generally, we find that the contributions from excited axial-vector mesons are more important than excited pions, corresponding to the fact that only the infinite tower of the former plays a role in satisfying the LSDCs (which is completely satisfied in the asymmetric MelnikovVainshtein case, and at the level of $81 \%$ in the symmetric case). Massive pions already contribute in the HW1 and HW3 models in the chiral limit; away from the chiral limit, their importance is not increased, despite the different asymptotic behavior of their summed contribution in the HW1m and HW3m cases, where one has a logarithmic

\footnotetext{
${ }^{10}$ The model of Refs. [24,25] respects the known experimental constraints, but it was constructed such that the longitudinal SDCs are saturated by excited pseudoscalars instead of axialvector mesons.
} 
TABLE II. Partial sums of the contributions of pions and of axial-vector mesons to $a_{\mu}$ in units of $10^{-11}$, where $a_{\mu(L)}$ denotes the longitudinal contribution only. Here, $\pi$ and $a_{1}$ refer to the entire tower of pions and $a_{1}$ mesons, $\pi^{*}$ only to the heavy pions, and $a_{1}^{*}$ only to excited axial-vector mesons. A and $\mathrm{P}^{*}$ refer to a whole $U\left(N_{f}=3\right)$ multiplet of axial-vector mesons and excited pseudoscalars, where the contributions from the former are split into longitudinal $(L)$ and transverse $(T)$ parts. In the present flavor-symmetric case, $a_{\mu}^{\mathrm{A}} \equiv 4 a_{\mu}^{a_{1}}$ and $a_{\mu}^{\mathrm{P}^{*}} \equiv 4 a_{\mu}^{\pi^{*}}$.

\begin{tabular}{|c|c|c|c|c|c|c|c|c|}
\hline Model & $a_{\mu}^{\pi^{0}}$ & $a_{\mu}^{\pi}$ & $a_{\mu}^{\pi \cup a_{1}}$ & $a_{\mu(L)}^{\pi^{*} \cup a_{1}}$ & $a_{\mu(L)}^{\pi^{*} \cup a_{1}^{*}}$ & $a_{\mu}^{\mathrm{P}^{*}}$ & $a_{\mu}^{\mathrm{A}}[L+T]$ & $a_{\mu}^{\mathrm{A}+\mathrm{P}^{*}}$ \\
\hline HW1 & 65.2 & 66.1 & 76.2 & 6.6 & 2.2 & 3.5 & $40.6[23.2+17.4]$ & 44.0 \\
\hline HW1m & 66.0 & 66.8 & 77.0 & 6.7 & 2.2 & 3.5 & $40.8[23.3+17.5]$ & 44.3 \\
\hline $\mathrm{HW} 1 \mathrm{~m}^{\prime}$ & 64.3 & 66.1 & 77.0 & 8.1 & 3.7 & 7.2 & $43.3[25.0+18.3]$ & 50.5 \\
\hline HW3m & 66.6 & 67.4 & 77.4 & 6.5 & 1.9 & 3.4 & $39.9[22.7+17.2]$ & 43.3 \\
\hline HW $3 \mathrm{~m}^{\prime}$ & 66.0 & 67.5 & 77.8 & 7.4 & 2.7 & 6.1 & $41.2[23.5+17.7]$ & 47.3 \\
\hline
\end{tabular}

enhancement of the TFFs. Correspondingly, the contributions of the axial-vector tower are not reduced; in fact, they tend to be higher with nonzero quark masses.

\section{E. Discussion}

Since we have seen above that the holographic results for the low-energy observables $F_{\pi_{2} \gamma \gamma}$ and $A_{1}(0,0)$, the latter determining the equivalent two-photon rate of the lightest axial-vector meson, are larger than indicated by experiments, the corresponding holographic results may perhaps be viewed as upper limits. In the following, we investigate whether this situation improves when one tries to accommodate corrections to the high-energy behavior. In fact, the holographic HW models we have considered here have no running coupling constant; the TFFs reach their asymptotic UV limits somewhat too quickly.

In order to derive more plausible extrapolations to real QCD, we have considered a reduction of the value $g_{5}^{2}$ by $10 \%$ and by $15 \%$. This brings the asymptotic behavior of the TFFs down by amounts that are roughly consistent with perturbative corrections to the leading-order $\mathrm{pQCD}$ results at moderately high $Q^{2}$ values $[80,81]$. At the same time, the right-hand side of Eq. (14) is increased by a similar amount, which is consistent with the next-to-leading-order terms in this expression [82].

With 10\% reduction, the HW model results for the pion TFF also get closer to the central result of the dispersive approach at all energies, while with $15 \%$ they are generally somewhat lower. In Table III, we have listed the reduction

TABLE III. Reduction factors $R$ in pseudoscalar and axialvector contributions to $a_{\mu}$ as estimated from the chiral HW model when the asymptotic LO pQCD constraints for TFFs are satisfied only at the levels of $90 \%$ or $85 \%$. $P$ and $A$ refer to the whole tower of pseudoscalar and axial-vector mesons; $P_{1}$ and $A_{1}$ refer to the ground-state modes.

\begin{tabular}{lcccc}
\hline \hline$\frac{g_{5}^{2} N_{c}}{12 \pi^{2}}$ & $R\left(A(0,0)_{n=1}\right)$ & $R\left(a_{\mu}^{P_{1}, P}\right)$ & $R\left(a_{\mu}^{\mathrm{A}_{1}}\right)$ & $R\left(a_{\mu}^{\mathrm{A}}\right)$ \\
\hline 0.90 & 0.93 & 0.96 & 0.91 & 0.95 \\
0.85 & 0.90 & 0.94 & 0.87 & 0.92 \\
\hline \hline
\end{tabular}

factors resulting for $A_{1}(0,0)$ and various $a_{\mu}$ contributions in the chiral HW1 model, which we assume to be a good approximation in general. Applying the stronger reduction factors to the minimum values of the results of the massive HW models, we obtain the range

$$
\begin{aligned}
a_{\mu}^{\pi^{0}} & =\left(R\left(a_{\mu}^{P_{1}}\right)_{0.85} \times 64.3 \ldots 66.6\right) \times 10^{-11} \\
& =(60.5 \ldots 66.6) \times 10^{-11}
\end{aligned}
$$

in remarkable agreement with recent evaluations using the data-driven dispersive approach [22], where $a_{\mu}^{\pi^{0}}=$ $62.6_{-2.5}^{+3.0} \times 10^{-11}$, which has also been backed up by lattice QCD [22]. Doing the same for excited pseudoscalars and axial-vector mesons, we obtain the following ranges as our predictions for the contributions of excited pseudoscalars and axial-vector mesons:

$$
\begin{aligned}
a_{\mu}^{\mathrm{P}^{*}} & \equiv 4 a_{\mu}^{\pi^{*}}=(3.2 \ldots 7.2) \times 10^{-11}, \\
a_{\mu(L)}^{\mathrm{A}} & =(20.8 \ldots 25.0) \times 10^{-11}, \\
a_{\mu}^{\mathrm{A}} & =(36.6 \ldots 43.3) \times 10^{-11}, \\
a_{\mu}^{\mathrm{A}+\mathrm{P}^{*}} & =(39.8 \ldots 50.5) \times 10^{-11} .
\end{aligned}
$$

The latter result could be compared to the white paper [4] values attributed to the axial sector and contributions related to the $\mathrm{SDC}, a_{\mu}^{\mathrm{WP}, \text { axials }}=6(6) \times 10^{-11}$ and $a_{\mu}^{\mathrm{WP}, \mathrm{SDC}}=$ $15(10) \times 10^{-11}$, which with linearly added errors gives $21(16) \times 10^{-11}$, which is significantly smaller.

In Ref. [46], a model-independent estimate of the effects of the longitudinal short-distance constraints on the HLBL contribution to $a_{\mu}$ has been proposed, with the result $\Delta a_{\mu}^{(3)}=2.6(1.5) \times 10^{-11}$ for the isovector sector. In Table II, we have also listed the results for the longitudinal part of the contributions from the excited pions and the $a_{1}$ tower. Extending the range of the holographic results by the above reduction of the lower end, we obtain $a_{\mu(L)}^{\pi^{*} \cup a_{1}}=(6.0 \ldots 8.1) \times 10^{-11}$, which is significantly higher. However, excluding the ground-state axial-vector meson, 
whose mass is close to the matching scale used in Ref. [46] and which (like any single excitation) does not contribute to the asymptotic value of the TFFs, we just have $a_{\mu(L)}^{\pi^{*} \cup a_{1}^{*}}=$ $(1.7 \ldots 3.7) \times 10^{-11}$, in perfect agreement with $\Delta a_{\mu}^{(3)}$ of Ref. [46]. Including singlet and octet contributions, Ref. [46] has estimated $\Delta a_{\mu}^{(0)+(3)+(8)}=9.1(5.0) \times 10^{-11}$, which is 3.5 times the $\Delta a_{\mu}^{(3)}$ result. In our flavor-symmetric models, we would have to multiply our results by a factor of 4 , giving $(6.9 \ldots 15.0) \times 10^{-11}$, which is still agreeing well. It would be interesting to revisit the additional study in Ref. [46], where the HW2 results for the lightest axialvector meson were included and a result was found that exceeded the contributions of the remaining tower.

Another method to estimate the effects of the LSDC has been used in Refs. [24,25], where a Regge model of excited pseudoscalars has been tuned to reproduce the LSDC. Although we have found in the holographic models that away from the chiral limit also, it is the axial-vector mesons that are alone responsible for the LSDC, the recently updated estimate obtained in Ref. [47], $\Delta a_{\mu}^{\mathrm{LSDC}}=13(5)$, is fully consistent with our conclusions, as illustrated in Fig. 10.

The most important contribution missing in previous evaluations of the HLBL piece of $a_{\mu}$, if the holographic HW models are to be trusted, are those from the groundstate axial-vector mesons. With the reduction of $g_{5}$, the lowenergy end of the axial-vector TFF also gets modified appreciably, expanding our range of predictions to

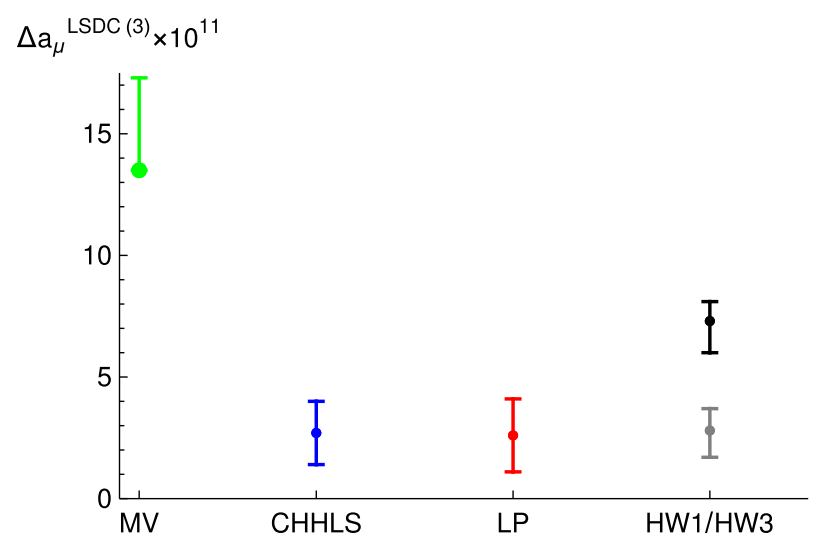

FIG. 10. Isovector part of the longitudinal contributions beyond the pion pole. The original estimate of Melnikov-Vainshtein [8] (MV) of $13.5 \times 10^{-11}$ (green dot) translates to $17.5 \times 10^{-11}$ with current input according to Refs. [24,25]. The estimate obtained by the latter, who have constructed a Regge model for excited pseudoscalars to reproduce the MV-SDC, is shown in blue and labelled CHHLS. The estimate of Lüdtke and Procura [46] (LP) is plotted in red. The spread of results obtained in the HW1/HW3 models is shown in black; the gray version is without the contribution from the ground-state $a_{1}$ meson. (The center of the error bar is the center of the uncorrected results, and the errors are enlarged downwards according to the $85 \%$ reduction of $g_{5}^{2}$.).
$|A(0,0)|_{n=1}=(17.3 \ldots 21.3) \mathrm{GeV}^{-2}$, which now has overlap with the experimental values quoted above in Sec. V C. The lowest value (which also fits the experimental data best) is in fact obtained in the $\mathrm{HW} 1 \mathrm{~m}^{\prime}$ model, where the mass of $a_{1}(1260)$ can be fitted, yielding ${ }^{11}$ $a_{\mu}^{\mathrm{A}_{1}}=25.9 \times 10^{-11}$. In this model, however, the $n=2$ axial-vector TFF has a larger value than in the other models, and its contribution to $a_{\mu}$ is also fairly large, so that the second-lightest axial-vector multiplet alone contributes another $a_{\mu}^{\mathrm{A}_{2}}=7.5 \times 10^{-11}$, despite having a mass much higher than those of established excited axial-vector mesons. As we have already remarked, the total contribution of the axial-vector tower is the largest of all HW models (see Table II). With maximal reduction of $g_{5}^{2}$, it still yields $a_{\mu}^{\mathrm{A}}=40 \times 10^{-11}$, coinciding with the central value of the range given in Eq. (62).

All in all, the holographic HW models that we have considered here point to $\gtrsim 20 \times 10^{-11}$ of extra contributions in the HLBL part of $a_{\mu}$, compared to the axial-vector and SDC pieces in the white paper value [4] of $a_{\mu}^{\mathrm{HLBL}, \mathrm{WP}}=92(18) \times 10^{-11}$, chiefly due to the axial-vector meson contributions. Such sizable upwards corrections are in fact compatible with the recent complete lattice calculation [28] with comparable errors, which obtained $a_{\mu}^{\mathrm{HLBL}, \text { lattice }}=106.8(14.7) \times 10^{-11}$.

\section{CONCLUSION}

In this paper, we have studied various AdS/QCD models with a hard wall and with a bifundamental scalar, which permits us to introduce finite quark masses, and thus to extend our previous work on hadronic light-by-light contributions in holographic QCD away from the chiral limit. In the latter, it was shown in Refs. [34,35] that summation of the contributions of the infinite tower of axial-vector mesons changes the asymptotic behavior of the HLBL scattering amplitude of individual contributions precisely such that the Melnikov-Vainshtein LSDC is satisfied. By contrast, in Refs. [24,25], a Regge model of excited pseudoscalars has been constructed to achieve the same. Turning on finite quark masses, we found that in the holographic models, the infinite tower of excited pseudoscalars, which is already present in the chiral limit and in fact has nonvanishing two-photon couplings despite vanishing decay constants, couples to the axial anomaly and then can lead to a certain enhancement of their contribution to the asymptotic HLBL amplitude, but never enough to contribute to the leading terms of the LSDCs. With the standard choice of the holographic mass of the bifundamental scalar, which determines the scaling dimension of quark masses and chiral condensates, this enhancement is

\footnotetext{
${ }^{11}$ This is significantly larger than our previous "data-based" extrapolation in Ref. [34], which was using an experimental value for $A(0,0)$ that is lower than the ones discussed in Sec. V C.
} 
merely logarithmic; generalizations are possible where power-law enhancements also arise, but they are still below what is relevant for LSDCs at leading order.

We have also considered the numerical consequences of introducing finite quark masses on the results obtained previously in the chiral limit, for simplicity only in the flavor-symmetric limit so that the $\pi^{0}$ and the $a_{1}$ sector can be covered, leaving the $N_{f}=2+1$ case and also the consideration of the Witten-Veneziano mechanism for the $\mathrm{U}(1)_{A}$ anomaly to future work. Doing so, we have explored the two sets of boundary conditions that are possible in hard-wall models, and we have also considered the generalization of modified scaling dimensions of quark masses and chiral condensates proposed in Ref. [51], which permits us to fit either the mass of the first excited pion or the mass of the lowest axial-vector meson.

As displayed in Fig. 9, the massive HW models lead only to small (positive) changes in the contributions to $a_{\mu}$ from the $\pi^{0}$ and $a^{0}$ towers compared to the chiral HW1 model, when in the latter the physical pion mass is inserted manually in the pion propagator. Compared to experimental data, the two-photon couplings of the lowest axial-vector meson, which is responsible for the second-largest contribution besides the ground-state pion, is somewhat too large, but it becomes consistent with experimental constraints when the five-dimensional coupling is adjusted such that the LO pQCD values of TFFs are reduced by amounts corresponding to typical $\alpha_{s}$ corrections at moderately large energies. However, after such adjustments, the contributions from the axial-vector and excited pseudoscalar mesons $\left(\gtrsim 40 \times 10^{-11}\right)$ are also significantly larger than in the model calculations that have been used to assess their role and also the effect of SDCs in the white paper [4], where $a_{\mu}^{\text {axials }+\mathrm{SDC}}=21(16) \times 10^{-11}$.

\section{ACKNOWLEDGMENTS}

We would like to thank Hans Bijnens, Luigi Cappiello, Oscar Catà, Giancarlo D'Ambrosio, Gilberto Colangelo, Franziska Hagelstein, Martin Hoferichter, Jan Lüdtke, Jonas Mager, Massimiliano Procura, and Peter Stoffer for discussions. J. L. was supported by the FWF doctoral program Particles \& Interactions, Project No. W1252-N27.

\section{APPENDIX A: CHIRAL LIMIT AND GOR RELATION}

In the following, we give some details on the chiral limit $M_{q} \rightarrow 0$ of the massive HW models, including the generalization where $M_{X}^{2}$ is allowed to deviate from the standard choice $M_{X}^{2}=-3$. The connection to the chiral HW models with strictly $M_{q}=0$ is somewhat subtle, because the weight function $z / \beta(z)$ in the differential equation [Eq. (22)] for $y=\partial_{z} \phi / z$ is (more) singular at $\zeta \equiv z / z_{0}=0$ when $M_{q}=0$ :

$$
\frac{z}{\beta(z)} \sim \begin{cases}\frac{z_{0}}{g_{5}^{2} M_{q}^{2}} \zeta^{-1+2 \alpha} & \text { for } \zeta \rightarrow 0, M_{q} \neq 0 \\ \frac{1}{g_{5}^{2} \Sigma^{2} z_{0}^{\zeta}} \zeta^{-1-2 \alpha} & \text { for } \zeta \rightarrow 0, M_{q}=0\end{cases}
$$

where $\alpha=\sqrt{4+M_{X}^{2}} \in(0,2)$, with $\alpha=1$ for the standard choice $M_{X}^{2}=-3$.

The asymptotic behavior of the profile function $y_{S}(q, z)$ near the boundary $\zeta=0$ reads

$y_{S}(q, z) \sim \begin{cases}\frac{g_{5}^{2} M_{q}^{2}}{2-2 \alpha} \zeta^{2-2 \alpha}+a_{1}+a_{2} \zeta^{4-2 \alpha}+\cdots & \text { for } \alpha>1 \\ g_{5}^{2} M_{q}^{2} \ln \zeta+a_{1}+a_{2} \zeta^{2} \ln \zeta+\cdots & \text { for } \alpha=1, \\ a_{0}+\frac{g_{5}^{2} M_{q}^{2}}{2-2 \alpha} \zeta^{2-2 \alpha}+a_{2} \zeta^{2}+\cdots & \text { for } \alpha<1\end{cases}$

and that of normalizable modes is given by

$$
y_{n}=-g_{5} f_{\pi_{n}}+\left\{\begin{array}{ll}
c_{1} \zeta^{4-2 \alpha}+\cdots & \text { for } \alpha>1 \\
c_{1} \zeta^{2}+\cdots & \text { for } \alpha \leq 1
\end{array} .\right.
$$

The mode functions $\phi_{n}$ and $\pi_{n}$ vanish at the ultraviolet boundary according to

$\phi_{n}=-g_{5} f_{\pi_{n}} z^{2} / 2+\cdots, \quad \pi_{n}=-\frac{m_{n}^{2} f_{\pi_{n}} z_{0}^{2-2 \alpha}}{2 \alpha g_{5} M_{q}^{2}} z^{2 \alpha}+\cdots$.

When $M_{q} \ll \Sigma z_{0}^{2}$, the weight function $z / \beta(z)$ is concentrated at small $z$, where its would-be divergence is cut off by $M_{q}$. In this limit, one can approximate the normalization condition [Eq. (27)] by replacing $y_{n}^{2}$ with its boundary value $g_{5}^{2} f_{\pi_{n}}^{2}$ and the upper limit of the integral by infinity, yielding

$$
g_{5}^{2} f_{\pi_{n}}^{2} m_{n}^{2} \int_{0}^{\infty} d z \frac{z}{\beta(z)}=1,
$$

where

$$
\begin{aligned}
g_{5}^{2} \int_{0}^{\infty} d z \frac{z}{\beta(z)} & =z_{0}^{4} \int_{0}^{\infty} d \zeta \frac{\zeta^{3}}{\left(z_{0} M_{q} \zeta^{\Delta^{-}}+z_{0}^{3} \Sigma \zeta^{\Delta^{+}}\right)^{2}} \\
& =z_{0}^{4} \int_{0}^{\infty} d \zeta \frac{\zeta^{2 \alpha-1}}{\left(z_{0} M_{q}+z_{0}^{3} \Sigma \zeta^{2 \alpha}\right)^{2}}=\frac{1}{2 \alpha M_{q} \Sigma} .
\end{aligned}
$$

For sufficiently small $M_{q}$, we thus obtain

$$
f_{\pi_{n}}^{2} m_{n}^{2} \approx 2 \alpha M_{q} \Sigma
$$

For massive pions-i.e., for $n>1$ - where $m_{n}$ approaches a nonzero value in the chiral limit, this implies that $f_{\pi_{n}} \rightarrow 0$, so they decouple, while the lightest pion with 
$f_{\pi_{1}}=f_{\pi}$ gives rise to the Gell-Mann-Oakes-Renner relation $f_{\pi}^{2} m_{\pi}^{2}=2 M_{q} \Sigma_{q}$ for $\alpha=1$, while for $\alpha \neq 1$ one should perhaps rescale $M_{q}$ and $\Sigma$ before interpreting them as quark mass and condensate. (The scaling factor mentioned in footnote 2 drops out here.)

While $y_{1}$ always satisfies the boundary condition $\frac{z}{\beta} \partial_{z} y_{1}=0$ at $z=\epsilon$ with $z / \beta \sim z^{-1+2 \alpha}$ as $M_{q} \rightarrow 0$, it does not satisfy such a boundary condition with $\left.\beta(z)\right|_{M_{q}=0}$. From the point of view of the strictly chiral HW model, $y_{1}$ corresponds to a solution with different boundary conditions that pertain to those of profile functions $y_{S}$ (up to an overall factor). Nevertheless, it can still be normalized by Eq. (27), since with the help of the equations of motion, the divergent integral times the vanishing mass can be recast as

$m_{1}^{2} \int_{\epsilon}^{z_{0}} d z \frac{z}{\beta(z)} y_{1}^{2}=\int_{\epsilon}^{z_{0}} d z y_{1}\left[z-\partial_{z} \frac{z}{\beta(z)} \partial_{z}\right] y_{1}$.
The holographic wave function of the massless pion can be given in closed form as the appropriate linear combination of the two Bessel functions:

$$
z^{1+\alpha} I_{ \pm \frac{1+\alpha}{2+\alpha}}\left(\frac{g_{5} \Sigma z_{0}^{1-\alpha}}{2+\alpha} z^{2+\alpha}\right) .
$$

In the special case of the chiral HW1 model with standard $M_{X}^{2}=-3$, and thus $\alpha=1$, the result reads [52]

$$
y_{1}=N z^{2}\left(-I_{-\frac{2}{3}}\left(\eta z^{3}\right)+\frac{I_{-\frac{2}{3}}\left(\eta z_{0}^{3}\right)}{I_{\frac{2}{3}}\left(\eta z_{0}^{3}\right)} I_{\frac{2}{3}}\left(\eta z^{3}\right)\right),
$$

with $\eta=g_{5} \Sigma / 3$ and $N^{2}=g_{5}^{2} \Sigma^{2} \Gamma\left(\frac{1}{3}\right) \Gamma\left(\frac{2}{3}\right) I_{\frac{2}{3}}\left(\eta z_{0}^{3}\right) / I_{-\frac{2}{3}}\left(\eta z_{0}^{3}\right)$.

\section{APPENDIX B: EFFECTS OF REDUCING $\boldsymbol{g}_{5}^{2}$}

Table IV shows the changes brought about by a reduction of $g_{5}^{2}$ by $10 \%$ (HW1-) and by $15 \%$ (HW1 - -) in the chiral HW1 model.

\begin{tabular}{|c|c|c|c|c|c|c|c|c|c|c|}
\hline Model & PS & $n=1$ & $n=2$ & $n=3$ & AV & $n=1$ & $n=2$ & $n=3$ & $n=4$ & $n=5$ \\
\hline HW1 - chiral & $m_{\pi_{n}}[\mathrm{MeV}]$ & 0 や 135 & 1849 & 2847 & $m_{\mathrm{A}_{0}}[\mathrm{MeV}]$ & 1295 & 2065 & 2944 & 3906 & 4893 \\
\hline \multirow[t]{3}{*}{$M_{X}^{2}=-3$} & $f_{\pi_{n}}^{n}[\mathrm{MeV}]$ & $92.4 *$ & 0 & 0 & $F_{\mathrm{A}_{n}} / m_{\mathrm{A}_{n}}[\mathrm{MeV}]$ & 166 & 205 & 264 & 311 & 350 \\
\hline & $F_{\pi_{n} \gamma \gamma}\left[\mathrm{GeV}^{-1}\right]$ & 0.274 & -0.209 & 0.161 & $A(0,0)\left[\mathrm{GeV}^{-2}\right]$ & -19.65 & -4.49 & 0.390 & -2.06 & 0.353 \\
\hline & $a_{\mu}^{\pi_{n}} \times 10^{11}$ & 62.7 & 0.8 & 0.1 & $a_{\mu}^{\mathrm{A}_{n}} \times 10^{11}$ & 28.7 & 5.6 & 1.7 & 1.1 & 0.4 \\
\hline HW1 - - chiral & $m_{\pi_{n}}[\mathrm{MeV}]$ & $0 や 135$ & 1827 & 2830 & $m_{\mathrm{A}_{n}}[\mathrm{MeV}]$ & 1255 & 2027 & 2923 & 3892 & 4882 \\
\hline \multirow[t]{3}{*}{$M_{X}^{2}=-3$} & $f_{\pi_{n}}[\mathrm{MeV}]$ & $92.4^{*}$ & 0 & 0 & $F_{\mathrm{A}_{n}} / m_{\mathrm{A}_{n}}[\mathrm{MeV}]$ & 161 & 206 & 265 & 311 & 351 \\
\hline & $F_{\pi_{n} \gamma \gamma}\left[\mathrm{GeV}^{-1}\right]$ & 0.274 & -0.213 & 0.166 & $A(0,0)\left[\mathrm{GeV}^{-2}\right]$ & -18.90 & -5.13 & 0.406 & -2.00 & 0.332 \\
\hline & $a_{\mu}^{\pi_{n}} \times 10^{11}$ & 61.4 & 0.8 & 0.2 & $a_{\mu}^{\mathrm{A}_{n}} \times 10^{11}$ & 27.3 & 5.9 & 1.7 & 1.1 & 0.4 \\
\hline
\end{tabular}

TABLE IV. HW1 chiral models with reduced UV asymptotics. HW $1-$ and HW $1--$ have $g^{2} N_{c} / 12 \pi^{2}=0.90$ and 0.85 , respectively.

[1] B. Abi et al. (Muon $g-2$ Collaboration), Measurement of the Positive Muon Anomalous Magnetic Moment to 0.46 ppm, Phys. Rev. Lett. 126, 141801 (2021).

[2] G. W. Bennett et al. (Muon $g-2$ Collaboration), Final report of the muon E821 anomalous magnetic moment measurement at BNL, Phys. Rev. D 73, 072003 (2006).

[3] F. Jegerlehner, The Anomalous Magnetic Moment of the Muon, Second Edition, Springer Tracts Mod. Phys. 274, 1 (2017).

[4] T. Aoyama et al., The anomalous magnetic moment of the muon in the Standard Model, Phys. Rep. 887, 1 (2020).

[5] T. Aoyama, M. Hayakawa, T. Kinoshita, and M. Nio, Complete Tenth-Order QED Contribution to the Muon $g-2$, Phys. Rev. Lett. 109, 111808 (2012); T. Aoyama, T. Kinoshita, and M. Nio, Revised and improved value of the QED tenth-order electron anomalous magnetic moment,
Phys. Rev. D 97, 036001 (2018); Theory of the anomalous magnetic moment of the electron, Atoms 7, 28 (2019).

[6] A. Czarnecki, W. J. Marciano, and A. Vainshtein, Refinements in electroweak contributions to the muon anomalous magnetic moment, Phys. Rev. D 67, 073006 (2003); Erratum, Phys. Rev. D 73, 119901 (2006).

[7] C. Gnendiger, D. Stöckinger, and H. Stöckinger-Kim, The electroweak contributions to $(g-2)_{\mu}$ after the Higgs boson mass measurement, Phys. Rev. D 88, 053005 (2013).

[8] K. Melnikov and A. Vainshtein, Hadronic light-by-light scattering contribution to the muon anomalous magnetic moment revisited, Phys. Rev. D 70, 113006 (2004).

[9] J. Prades, E. de Rafael, and A. Vainshtein, The hadronic light-by-light scattering contribution to the muon and electron anomalous magnetic moments, Adv. Ser. Dir. High Energy Phys. 20, 303 (2009). 
[10] A. Kurz, T. Liu, P. Marquard, and M. Steinhauser, Hadronic contribution to the muon anomalous magnetic moment to next-to-next-to-leading order, Phys. Lett. B 734, 144 (2014).

[11] G. Colangelo, M. Hoferichter, A. Nyffeler, M. Passera, and P. Stoffer, Remarks on higher-order hadronic corrections to the muon $g-2$, Phys. Lett. B 735, 90 (2014).

[12] V. Pauk and M. Vanderhaeghen, Single meson contributions to the muon's anomalous magnetic moment, Eur. Phys. J. C 74, 3008 (2014).

[13] M. Davier, A. Hoecker, B. Malaescu, and Z. Zhang, Reevaluation of the hadronic vacuum polarisation contributions to the Standard Model predictions of the muon $g-2$ and $\alpha\left(m_{Z}^{2}\right)$ using newest hadronic cross-section data, Eur. Phys. J. C 77, 827 (2017).

[14] P. Masjuan and P. Sanchez-Puertas, Pseudoscalar-pole contribution to the $\left(g_{\mu}-2\right)$ : A rational approach, Phys. Rev. D 95, 054026 (2017).

[15] G. Colangelo, M. Hoferichter, M. Procura, and P. Stoffer, Dispersion relation for hadronic light-by-light scattering: Two-pion contributions, J. High Energy Phys. 04 (2017) 161.

[16] A. Keshavarzi, D. Nomura, and T. Teubner, Muon $g-2$ and $\alpha\left(M_{Z}^{2}\right)$ : A new data-based analysis, Phys. Rev. D 97, 114025 (2018).

[17] G. Colangelo, M. Hoferichter, and P. Stoffer, Two-pion contribution to hadronic vacuum polarization, J. High Energy Phys. 02 (2019) 006.

[18] M. Hoferichter, B.-L. Hoid, and B. Kubis, Three-pion contribution to hadronic vacuum polarization, J. High Energy Phys. 08 (2019) 137.

[19] M. Davier, A. Hoecker, B. Malaescu, and Z. Zhang, A new evaluation of the hadronic vacuum polarisation contributions to the muon anomalous magnetic moment and to $\alpha\left(m_{Z}^{2}\right)$, Eur. Phys. J. C 80, 241 (2020).

[20] A. Keshavarzi, D. Nomura, and T. Teubner, $g-2$ of charged leptons, $\alpha\left(M_{Z}^{2}\right)$, and the hyperfine splitting of muonium, Phys. Rev. D 101, 014029 (2020).

[21] M. Hoferichter, B.-L. Hoid, B. Kubis, S. Leupold, and S. P. Schneider, Pion-Pole Contribution to Hadronic Light-byLight Scattering in the Anomalous Magnetic Moment of the Muon, Phys. Rev. Lett. 121, 112002 (2018); Dispersion relation for hadronic light-by-light scattering: Pion pole, J. High Energy Phys. 10 (2018) 141.

[22] A. Gérardin, H. B. Meyer, and A. Nyffeler, Lattice calculation of the pion transition form factor with $N_{f}=2+1$ Wilson quarks, Phys. Rev. D 100, 034520 (2019).

[23] J. Bijnens, N. Hermansson-Truedsson, and A. RodríguezSánchez, Short-distance constraints for the HLbL contribution to the muon anomalous magnetic moment, Phys. Lett. B 798, 134994 (2019).

[24] G. Colangelo, F. Hagelstein, M. Hoferichter, L. Laub, and P. Stoffer, Short-distance constraints on hadronic light-by-light scattering in the anomalous magnetic moment of the muon, Phys. Rev. D 101, 051501 (2020).

[25] G. Colangelo, F. Hagelstein, M. Hoferichter, L. Laub, and P. Stoffer, Longitudinal short-distance constraints for the hadronic light-by-light contribution to $(g-2)_{\mu}$ with large- $N_{c}$ Regge models, J. High Energy Phys. 03 (2020) 101.
[26] I. Danilkin, C. F. Redmer, and M. Vanderhaeghen, The hadronic light-by-light contribution to the muon's anomalous magnetic moment, Prog. Part. Nucl. Phys. 107, 20 (2019).

[27] T. Blum, N. Christ, M. Hayakawa, T. Izubuchi, L. Jin, C. Jung, and C. Lehner, Hadronic Light-by-Light Scattering Contribution to the Muon Anomalous Magnetic Moment from Lattice QCD, Phys. Rev. Lett. 124, 132002 (2020).

[28] E.-H. Chao, R. J. Hudspith, A. Gérardin, J. R. Green, H. B. Meyer, and K. Ottnad, Hadronic light-by-light contribution to $(g-2)_{\mu}$ from lattice QCD: A complete calculation, Eur. Phys. J. C 81, 651 (2021).

[29] S. Borsanyi et al., Leading hadronic contribution to the muon magnetic moment from lattice QCD, Nature (London) 593, 51 (2021).

[30] A. Crivellin, M. Hoferichter, C. A. Manzari, and M. Montull, Hadronic Vacuum Polarization: $(g-2)_{\mu}$ versus Global Electroweak Fits, Phys. Rev. Lett. 125, 091801 (2020).

[31] A. Keshavarzi, W. J. Marciano, M. Passera, and A. Sirlin, Muon $g-2$ and $\Delta \alpha$ connection, Phys. Rev. D 102, 033002 (2020).

[32] G. Colangelo, M. Hoferichter, and P. Stoffer, Constraints on the two-pion contribution to hadronic vacuum polarization, Phys. Lett. B 814, 136073 (2021).

[33] M. Abe et al., A new approach for measuring the muon anomalous magnetic moment and electric dipole moment, Prog. Theor. Exp. Phys. (2019), $053 \mathrm{C} 02$.

[34] J. Leutgeb and A. Rebhan, Axial vector transition form factors in holographic QCD and their contribution to the anomalous magnetic moment of the muon, Phys. Rev. D 101, 114015 (2020).

[35] L. Cappiello, O. Catà, G. D’Ambrosio, D. Greynat, and A. Iyer, Axial-vector and pseudoscalar mesons in the hadronic light-by-light contribution to the muon $(g-2)$, Phys. Rev. D 102, 016009 (2020).

[36] M. Knecht, On some short-distance properties of the fourthrank hadronic vacuum polarization tensor and the anomalous magnetic moment of the muon, J. High Energy Phys. 08 (2020) 056.

[37] P. Masjuan, P. Roig, and P. Sanchez-Puertas, The interplay of transverse degrees of freedom and axial-vector mesons with short-distance constraints in $g-2$, arXiv:2005.11761.

[38] J. Erlich, E. Katz, D. T. Son, and M. A. Stephanov, QCD and a Holographic Model of Hadrons, Phys. Rev. Lett. 95, 261602 (2005).

[39] L. Da Rold and A. Pomarol, Chiral symmetry breaking from five-dimensional spaces, Nucl. Phys. B721, 79 (2005); The scalar and pseudoscalar sector in a five-dimensional approach to chiral symmetry breaking, J. High Energy Phys. 01 (2006) 157.

[40] L. Cappiello, O. Cata, and G. D'Ambrosio, The hadronic light by light contribution to the $(g-2)_{\mu}$ with holographic models of QCD, Phys. Rev. D 83, 093006 (2011).

[41] J. Leutgeb, J. Mager, and A. Rebhan, Pseudoscalar transition form factors and the hadronic light-by-light contribution to the anomalous magnetic moment of the muon from holographic QCD, Phys. Rev. D 100, 094038 (2019); Erratum, Phys. Rev. D 104, 059903 (2019).

[42] P. Achard et al. (L3 Collaboration), $f_{1}$ (1285) formation in two-photon collisions at LEP, Phys. Lett. B 526, 269 (2002). 
[43] K. Ghoroku, N. Maru, M. Tachibana, and M. Yahiro, Holographic model for hadrons in deformed $\mathrm{AdS}_{5}$ background, Phys. Lett. B 633, 602 (2006).

[44] A. Karch, E. Katz, D. T. Son, and M. A. Stephanov, Linear confinement and AdS/QCD, Phys. Rev. D 74, 015005 (2006).

[45] U. Gürsoy and E. Kiritsis, Exploring improved holographic theories for QCD: Part I, J. High Energy Phys. 02 (2008) 032; U. Gürsoy, E. Kiritsis, and F. Nitti, Exploring improved holographic theories for QCD: Part II, J. High Energy Phys. 02 (2008) 019.

[46] J. Lüdtke and M. Procura, Effects of longitudinal short-distance constraints on the hadronic light-by-light contribution to the muon $g-2$, Eur. Phys. J. C 80, 1108 (2020).

[47] G. Colangelo, F. Hagelstein, M. Hoferichter, L. Laub, and P. Stoffer, Short-distance constraints for the longitudinal component of the hadronic light-by-light amplitude: An update, Eur. Phys. J. C 81, 702 (2021).

[48] J. Hirn and V. Sanz, Interpolating between low and high energy QCD via a 5D Yang-Mills model, J. High Energy Phys. 12 (2005) 030.

[49] T. Sakai and S. Sugimoto, Low energy hadron physics in holographic QCD, Prog. Theor. Phys. 113, 843 (2005).

[50] T. Sakai and S. Sugimoto, More on a holographic dual of QCD, Prog. Theor. Phys. 114, 1083 (2005).

[51] O. Domènech, G. Panico, and A. Wulzer, Massive pions, anomalies and baryons in holographic QCD, Nucl. Phys. A853, 97 (2011).

[52] H. R. Grigoryan and A. V. Radyushkin, Pion form-factor in chiral limit of hard-wall AdS/QCD model, Phys. Rev. D 76, 115007 (2007).

[53] Z. Abidin and C. E. Carlson, Strange hadrons and kaon-topion transition form factors from holography, Phys. Rev. D 80, 115010 (2009).

[54] A. Cherman, T. D. Cohen, and E. S. Werbos, The chiral condensate in holographic models of QCD, Phys. Rev. C 79, 045203 (2009).

[55] A. Gorsky and A. Krikun, Magnetic susceptibility of the quark condensate via holography, Phys. Rev. D 79, 086015 (2009).

[56] H. J. Kwee and R. F. Lebed, Pion form-factors in holographic QCD, J. High Energy Phys. 01 (2008) 027.

[57] D. T. Son and N. Yamamoto, Holography and anomaly matching for resonances, arXiv:1010.0718.

[58] H. R. Grigoryan and A. V. Radyushkin, Anomalous form factor of the neutral pion in extended AdS/QCD model with Chern-Simons term, Phys. Rev. D 77, 115024 (2008).

[59] H. R. Grigoryan and A. V. Radyushkin, Pion in the holographic model with 5D Yang-Mills fields, Phys. Rev. D 78, 115008 (2008).

[60] D. K. Hong and D. Kim, Pseudo scalar contributions to light-by-light correction of muon $g-2$ in AdS/QCD, Phys. Lett. B 680, 480 (2009).

[61] G. P. Lepage and S. J. Brodsky, Exclusive processes in quantum chromodynamics: Evolution equations for hadronic wave functions and the form-factors of mesons, Phys. Lett. 87B, 359 (1979); Exclusive processes in perturbative quantum chromodynamics, Phys. Rev. D 22, 2157 (1980);
S. J. Brodsky and G. P. Lepage, Large-angle two-photon exclusive channels in quantum chromodynamics, Phys. Rev. D 24, 1808 (1981).

[62] A. V. Efremov and A. V. Radyushkin, Factorization and asymptotical behavior of pion form-factor in QCD, Phys. Lett. 94B, 245 (1980).

[63] M. Hoferichter and P. Stoffer, Asymptotic behavior of meson transition form factors, J. High Energy Phys. 05 (2020) 159.

[64] V. A. Novikov, M. A. Shifman, A. I. Vainshtein, M. B. Voloshin, and V.I. Zakharov, Use and misuse of QCD sum rules, factorization and related topics, Nucl. Phys. B237, 525 (1984).

[65] L. D. Landau, On the angular momentum of a system of two photons, Dokl. Akad. Nauk Ser. Fiz. 60, 207 (1948).

[66] C.-N. Yang, Selection rules for the dematerialization of a particle into two photons, Phys. Rev. 77, 242 (1950).

[67] V. Pascalutsa, V. Pauk, and M. Vanderhaeghen, Light-bylight scattering sum rules constraining meson transition form factors, Phys. Rev. D 85, 116001 (2012).

[68] P. Roig and P. Sanchez-Puertas, Axial-vector exchange contribution to the hadronic light-by-light piece of the muon anomalous magnetic moment, Phys. Rev. D 101, 074019 (2020).

[69] M. Zanke, M. Hoferichter, and B. Kubis, On the transition form factors of the axial-vector resonance $f_{1}(1285)$ and its decay into $e^{+} e^{-}$, J. High Energy Phys. 07 (2021) 106.

[70] G. Colangelo, M. Hoferichter, M. Procura, and P. Stoffer, Dispersion relation for hadronic light-by-light scattering: Theoretical foundations, J. High Energy Phys. 09 (2015) 074.

[71] A. Vainshtein, Perturbative and nonperturbative renormalization of anomalous quark triangles, Phys. Lett. B 569, 187 (2003).

[72] P. A. Zyla et al., Review of particle physics, Prog. Theor. Exp. Phys. (2020), 083C01, 2021 updates available on-line.

[73] M. Diehl and G. Hiller, New ways to explore factorization in $b$ decays, J. High Energy Phys. 06 (2001) 067.

[74] K. Maltman and J. Kambor, Decay constants, light quark masses and quark mass bounds from light quark pseudoscalar sum rules, Phys. Rev. D 65, 074013 (2002).

[75] V. Elias, A. Fariborz, M. A. Samuel, F. Shi, and T. G. Steele, Beyond the narrow resonance approximation: Decay constant and width of the first pion excitation state, Phys. Lett. B 412, 131 (1997).

[76] A. L. Kataev, N. V. Krasnikov, and A. A. Pivovarov, The use of the finite energetic sum rules for the calculation of the light quark masses, Phys. Lett. 123B, 93 (1983).

[77] G. Ecker, J. Gasser, A. Pich, and E. de Rafael, The role of resonances in chiral perturbation theory, Nucl. Phys. B321, 311 (1989); G. Ecker, J. Gasser, H. Leutwyler, A. Pich, and E. de Rafael, Chiral lagrangians for massive spin 1 fields, Phys. Lett. B 223, 425 (1989).

[78] K.-C. Yang, Light-cone distribution amplitudes of axialvector mesons, Nucl. Phys. B776, 187 (2007).

[79] G. A. Schuler, F. A. Berends, and R. van Gulik, Meson photon transition form-factors and resonance cross-sections in $e^{+} e^{-}$collisions, Nucl. Phys. B523, 423 (1998). 
[80] B. Melic, D. Mueller, and K. Passek-Kumericki, Next-tonext-to-leading prediction for the photon to pion transition form-factor, Phys. Rev. D 68, 014013 (2003).

[81] J. Bijnens, N. Hermansson-Truedsson, L. Laub, and A. Rodríguez-Sánchez, The two-loop perturbative correction to the $(g-2)_{\mu}$ HLbL at short distances, J. High Energy Phys. 04 (2021) 240.

[82] M. A. Shifman, A. I. Vainshtein, and V. I. Zakharov, QCD and resonance physics. Theoretical foundations, Nucl. Phys. B147, 385 (1979). 